\title{
Regularity of optimal transport maps on multiple products of spheres
}

\author{
Received November 19, 2010 and in revised form May 23, 2011
}

\begin{abstract}
This article addresses regularity of optimal transport maps for cost $=$ "squared distance" on Riemannian manifolds that are products of arbitrarily many round spheres with arbitrary sizes and dimensions. Such manifolds are known to be non-negatively cross-curved [KM2]. Under boundedness and non-vanishing assumptions on the transfered source and target densities we show that optimal maps stay away from the cut-locus (where the cost exhibits singularity), and obtain injectivity and continuity of optimal maps. Together with the result of Liu, Trudinger and Wang [LTW] this also implies higher regularity $\left(C^{1, \alpha} / C^{\infty}\right)$ of optimal maps for smoother $\left(C^{\alpha} / C^{\infty}\right)$ densities. These are the first global regularity results which we are aware of concerning optimal maps on Riemannian manifolds which possess some vanishing sectional curvatures, beside the totally flat case of $\mathbb{R}^{n}[\mathrm{Ca} 3]$ and its quotients [Co]. Moreover, such product manifolds have potential relevance in statistics (see [S]) and in statistical mechanics (where the state of a system consisting of many spins is classically modeled by a point in the phase space obtained by taking many products of spheres). For the proof we apply and extend the method developed in [FKM1], where we showed injectivity and continuity of optimal maps on domains in $\mathbb{R}^{n}$ for smooth non-negatively cross-curved cost. The major obstacle in the present paper is to deal with the non-trivial cut-locus and the presence of flat directions.
\end{abstract}

Keywords. Optimal transport, functional inequalities and Riemannian geometry

\section{Contents}

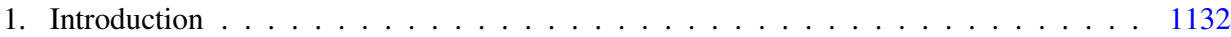

2. Notation and assumptions . . . . . . . . . . . . . . . . . . . . . . . 1134

3. Preliminary results . . . . . . . . . . . . . . . . . . . . . . . . 1139

4. An Alexandrov estimate: lower bound . . . . . . . . . . . . . . . . . . . . . . . . . . 1142

5. Stay-away property on multiple products of spheres . . . . . . . . . . . . . . . . 1145

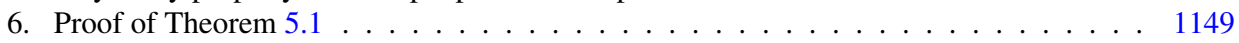

References . . . . . . . . . . . . . . . . . . . . . . . . . . . . 1164

A. Figalli: Department of Mathematics, The University of Texas at Austin, Austin, TX, USA; e-mail: figalli@math.utexas.edu

Y.-H. Kim: Department of Mathematics, University of British Columbia, Vancouver, BC, Canada; e-mail: yhkim@math.ubc.ca

R. J. McCann: Department of Mathematics, University of Toronto, Toronto, ON, Canada; e-mail: mccann@math.toronto.edu 


\section{Introduction}

Let $M$ and $\bar{M}$ be $n$-dimensional complete Riemannian manifolds, and let $\mu=\rho \operatorname{vol}_{M}$ and $\nu=\bar{\rho} \operatorname{vol}_{\bar{M}}$ be two probability measures whose densities $\rho$ and $\bar{\rho}$ are bounded away from zero and infinity. Given a cost function $c: M \times \bar{M} \rightarrow \mathbb{R}$, the optimal transport problem with cost $c(x, y)$ consists in finding a transport map $T: M \rightarrow \bar{M}$ which sends $\mu$ onto $v$ and minimizes the transportation cost

$$
\int_{M} c(x, T(x)) d \mu(x)
$$

As shown by McCann [M] extending the result of Brenier [Br] on $\mathbb{R}^{n}$, if $M=\bar{M}$ and $c=\operatorname{dist}^{2} / 2$ then the optimal transport map (or simply optimal map) exists and is unique. More generally, the same result holds if the cost is semiconcave and satisfies the twist condition in Assumption 2.1 below (see for instance [V, Chapter 10]).

The optimal map $T$ is uniquely characterized by the relation $T(x) \in \partial^{c} \phi(x)$, where $\phi$ is a $c$-convex function (called potential) and $\partial^{c} \phi$ denotes its $c$-subdifferential (see Section 2 for the definitions). Furthermore, the fact that $\rho$ and $\bar{\rho}$ are bounded away from zero and infinity ensures the existence of a constant $\lambda>0$ such that the following Monge-Ampère type equation holds:

$$
\lambda|\Omega| \leq\left|\partial^{c} \phi(\Omega)\right| \leq \frac{1}{\lambda}|\Omega| \quad \forall \Omega \subset M \text { Borel, }
$$

where $\partial^{c} \phi(\Omega)=\bigcup_{x \in \Omega} \partial^{c} \phi(x)$. (See for instance [FKM1, Lemma 3.1].)

The aim of this paper is to investigate the regularity of optimal maps when $M=\bar{M}$ are a multiple product of spheres, i.e., $M=\bar{M}=\mathbb{S}_{r_{1}}^{n_{1}} \times \cdots \times \mathbb{S}_{r_{k}}^{n_{k}}$, and $c(x, y)=f(\operatorname{dist}(x, y))$ for some function $f$, including the case $f(t)=t^{2} / 2$ of distance squared cost. For $k=1$ and $f(t)=t^{2} / 2$, smoothness of optimal maps has been proved by Loeper [L2]. However, if $k>1$ the structure of the cut-locus (the singular set of the cost function) becomes more complicated, and due to the product structure, the manifold has both flat and positively curved directions, thus making the regularity issue much more delicate. Especially, the powerful Hölder regularity estimate of Loeper [L1] (see also [Li]) as well as the a priori estimates of Ma, Trudinger and Wang [MTW], which are successfully applied to positively curved manifolds as in [L2, KM2, LV, FR, DG, FRV], are not available any more in our setting. Our main results (Theorem 5.1 and Corollary 5.3) give the first global regularity results which we are aware of concerning optimal maps on non-flat Riemannian manifolds which allow vanishing sectional curvature. For completely flat manifolds (with $c=$ dist $^{2} / 2$ ) the regularity of optimal maps is known as it reduces to the regularity theory of the classical Monge-Ampère equation [D1, Ca1, Ca2, Ca3, U, Ca4, Co, D2, G].

To describe our result more precisely, first recall that in [MTW] Ma, Trudinger and Wang discovered a condition (A3) on the cost function, whose weaker variant (A3w) [TW] turned out to be both necessary [L1] and sufficient [TW] for regularity when the solution $\phi$ is known to be strictly $c$-convex and the cost function is smooth. When $M=$ $\bar{M}=\mathbb{S}_{r}^{n}$, the particular structure of the cut-locus (for every point $x$, its cut-locus Cut $(x)$ consists of its antipodal point) allowed Delanoë and Loeper [DL] to deduce that optimal 
maps stay away from the cut-locus, namely, $\partial^{c} \phi(x) \cap \operatorname{Cut}(x)=\emptyset$ for all $x \in M$ (see [L2, DG, KM1, KM1a] for alternative approaches). Loeper [L2] combined this observation with the fact that $c=\operatorname{dist}^{2} / 2$ satisfies (A3) to show regularity of optimal maps; for a simpler approach to continuity, see [KM1, KM1a]. His result has been extended to a variety of positively curved manifolds including the complex projective space [KM2] and perturbation of the real projective space [LV] and of the sphere [FR, DG, FRV]. In each of these cases (A3) holds, thus the strong Hölder regularity estimate of [L1] as well as the a priori estimate of [MTW] applies. Note that (A3) (resp. (A3w)) forces the sectional curvature to be positive (resp. non-negative) [L1], though the converse does not hold [K].

On multiple products of spheres, taking $c=\mathrm{dist}^{2} / 2$ leads to two main issues: first, only a degenerate strengthening of the weak Ma-Trudinger-Wang condition holds (the so-called non-negative cross-curvature condition in [KM1, KM2]), which although stronger than $(\mathbf{A 3 w})$ is not as useful as $(\mathbf{A 3})$ for proving regularity due to lack of powerful estimates (neither non-negative cross-curvature nor (A3) implies the other, though either one separately implies (A3w)). Moreover, the cut-locus now has a non-trivial structure, which makes it much more difficult to understand whether the stay-away property holds. In [FKM1] we showed strict $c$-convexity and $C^{1}$ regularity of $\phi$, or equivalently, injectivity and continuity of $T$, when the cost is smooth and (A3w) holds. Hence the only question left is whether $\partial^{c} \phi$ avoids the cut-locus or not.

In this paper we answer this question positively: by taking advantage of the fact that the cut-locus is given by the union of certain subproducts of spheres we prove in Theorem 5.1 the stay-away property that $\partial^{c} \phi(x) \cap \operatorname{Cut}(x)=\emptyset$ for all $x \in M$. By compactness, these two sets are separated by a uniform distance that depends on $\lambda$, but is independent of the particular choice of $\phi$ and $x$ (see Corollary 5.2). Once stay-away property is shown, one can localize the argument of [FKM1] to obtain injectivity and continuity of the optimal map; then higher regularity $\left(C^{2, \alpha} / C^{\infty}\right)$ of $\phi$, thus $C^{1, \alpha} / C^{\infty}$-regularity of $T$, follows from [LTW] when the densities are smooth $\left(C^{\alpha} / C^{\infty}\right)$ (see Corollary 5.3).

The multiple products of spheres is a model case for more general manifolds on which the cost $c$ satisfies the necessary conditions [L1, FRV] for regularity of optimal transport maps. The method we develop in this paper demonstrates one approach to handling complex singularities of the cost, especially the stay-away property of optimal maps. Moreover, a general Alexandrov type estimate (Lemma 4.1) is obtained which has applications beyond the products of spheres.

Our regularity result has potential relevance to statistics and statistical mechanics. For instance, recently T. Sei applied optimal transport theory for $c=\operatorname{dist}^{2}$ to directional statistics on the sphere. In his main result [S, Theorem 1], he needed the optimal map not to touch the cut-locus. Now, our stay-away property on multiple products of spheres $M$ (Theorem 5.1) states that all optimal maps, obtained by transporting densities bounded away from zero and infinity onto each other, satisfy this assumption. Hence, this provides a large family of $c$-convex potentials that could be used to create log-concave likelihood functions as in [S, Subsection 3.2], extending his theory to multiple products of spheres. Namely, as a direct consequence of [KM2, FKM2, S], on multiple products of spheres a convex combination $\phi=\sum_{i=1}^{k} s_{i} \phi_{i}, s_{i} \geq 0, \sum s_{i}=1$, of $c$-convex functions $\phi_{i}$ is again $c$-convex, thus a crucial requirement in Sei's theory is satisfied. If each $\phi_{i}$ is the 
$c$-potential of an optimal map between densities bounded away from zero and infinity, by Theorem 5.1 one sees $\partial^{c} \phi_{i}$ stay away from the cut-locus. One can then show that $\partial^{c} \phi$ also avoids the cut-locus, thus applying [S, Theorem 1] one obtains the log-concave Jacobian inequality for this convex combination. To see this, for example, observe that in the product of spheres the "domain of exponential map" is convex, ${ }^{1}$ and $\partial^{c} \phi$ satisfies $\partial^{c} \phi(x)=\exp _{x} \partial \phi(x)=\exp _{x}\left[\sum_{i} \partial \phi_{i}(x)\right]$ for $x \in M$ (see Lemma 2.7). Since each $\partial^{c} \phi_{i}$ stays away from the cut-locus, $\partial \phi_{i}(x)$ belongs to the domain of the exponential map, hence so does $\partial \phi(x)$, showing $\partial^{c} \phi(x) \cap \operatorname{Cut}(x)=\emptyset$.

Concerning statistical mechanics, let us recall that the state of a spin system is classically modeled as a point in the phase space $M$ obtained by taking many products of spheres. In such contexts, optimal transport may provide a useful change of variables. More precisely, if $\mu$ and $v$ are two smooth densities and $T$ denotes the optimal transport map from $\mu$ to $v$, then

$$
\int G(y) d \nu(y)=\int G(T(x)) d \mu(x),
$$

for all bounded measurable functions $G: M \rightarrow \mathbb{R}$. Then, if $\mu$ is a "nice" measure for which many statistical quantities are easily computable, one may hope to exploit some qualitative/quantitative properties of $T$ in order to estimate the integral $\int G(y) d v(y)$ by studying $\int G(T(x)) d \mu(x)$. We expect that regularity of optimal maps may play a crucial role in this direction. For instance, in Euclidean spaces this is already the case, as Caffarelli [Ca5] used regularity of optimal maps to show that suitable monotonicity and log-concavity properties of the densities imply monotonicity and contraction properties for the optimal map, from which correlation and momentum inequalities may be deduced.

Organization of the paper. Section 2 sets up the notation and assumptions used throughout the paper. In Section 3, a few useful preliminary results regarding convex sets and $c$ convex functions are listed. Section 4 is devoted to an Alexandrov type inequality which is one of the main tools in the proof of our main theorem. Until Section 4, we present the theory under rather general assumptions. However, from Section 5 we restrict to the multiple products of spheres. In Section 5 we state our main result about the stay-away property of optimal maps, and give a sketch of the proof. Moreover we explain how one can deduce regularity of optimal maps combining this theorem with the results in [FKM1] and [LTW]. Finally, the details of the proof of the stay-away property are given in Section 6 .

\section{Notation and assumptions}

In this section and the next we recall notation and results which will be useful later. Many of these results originated in or were inspired by the work of Ma, Trudinger, Wang [MTW] and Loeper [L1]. Though the present paper mainly concerns the Riemannian

\footnotetext{
1 Here and below, we use "domain of exponential map" as a synonym for "injectivity domain" (see Section 2).
} 
distance squared cost $c=\mathrm{dist}^{2} / 2$ on the product of round spheres, we will present our work in a rather general framework. This requires only a small additional effort and may prove useful for further development and applications of the theory.

Let $M, \bar{M}$ be $n$-dimensional complete Riemannian manifolds, and let $c(x, \bar{x})$ denote a cost function $c: M \times \bar{M} \rightarrow \mathbb{R}$. We will assume throughout that $c$ is semiconcave in both variables, i.e., in coordinate charts it can be written as the sum of a concave and a smooth function. Let us remark that since $\operatorname{dist}^{2}(x, y)$ is semiconcave on $M \times M$ (see for example [FF, Appendix B]), the above assumption is satisfied for instance by any cost function of the form $f(\operatorname{dist}(x, y))$ on $M \times M$, with $f: \mathbb{R} \rightarrow \mathbb{R}$ smooth, even, and strongly convex (meaning $f(d)=f(-d)$ and $f^{\prime \prime}(d)>0$ for all $d \geq 0$ ). Here and below, "smooth" is synonymous with $C^{\infty}$ (though $C^{4}$ would be enough for all our purposes).

As for $\bar{x}$ and $\bar{M}$, we use the "bar" notation to specify the second variable of the cost function. Also we write $\bar{c}(\bar{x}, x):=c(x, \bar{x})$. We denote by $D_{x}$ and $D_{\bar{x}}$ the differentials with respect to the $x$ and $\bar{x}$ variable respectively. (For instance, $D_{x} D_{\bar{x}} c\left(x_{0}, \bar{x}_{0}\right)$ denotes the mixed partial derivative of $c$ at $\left(x_{0}, \bar{x}_{0}\right)$.) Let $c$-Cut $(\bar{x})$ denote the $c$-cut-locus of $\bar{x} \in \bar{M}$, that is,

$$
c \text {-Cut }(\bar{x}):=\{x \in M \mid c \text { is not smooth in a neighborhood of }(x, \bar{x})\},
$$

and let $M(\bar{x})$ denote the $c$-injectivity locus $M \backslash c$-Cut $(\bar{x})$. Define $\bar{c}$-Cut $(x), \bar{M}(x)$ similarly. These sets are open.

Assumption 2.1 (twist). For each $(x, \bar{x}) \in M \times \bar{M}$, the maps $-D_{x} c(x, \cdot): \bar{M}(x) \rightarrow$ $T_{x}^{*} M$ and $-D_{\bar{x}} c(\cdot, \bar{x}): M(\bar{x}) \rightarrow T_{\bar{x}}^{*} \bar{M}$ are smooth embeddings (thus injective).

We remark that the above hypothesis from Levin [L] is equivalent to condition (A1) in [MTW, L1, KM1], which together with the semiconcavity of the cost ensures existence and uniqueness of optimal maps when the source measure is absolutely continuous with respect to the volume measure (see for instance [L, FF, F] or [V, Chapter 10]).

The domain of the c-exponential $\bar{M}^{*}(\bar{x})$ in $T_{\bar{x}}^{*} \bar{M}$ is defined as the image of $M(\bar{x})$ under the map $-D_{\bar{x}} c(x, \cdot)$, i.e.,

$$
\bar{M}^{*}(\bar{x}):=-D_{\bar{x}} c(M(\bar{x}), \bar{x}) \subset T_{\bar{x}}^{*} \bar{M} .
$$

Define $M^{*}(x)$ similarly.

As in [MTW, L1], we define the $c$-exponential maps $c$-Exp $\operatorname{Ex}_{x}: M^{*}(x) \subset T_{x}^{*} M \rightarrow \bar{M}$ and $\bar{c}-\operatorname{Exp}_{\bar{x}}: \bar{M}^{*}(\bar{x}) \subset T_{\bar{x}}^{*} \bar{M} \rightarrow M$ as the inverse maps of $-D_{x} c(x, \cdot)$ and $-D_{\bar{x}} c(\cdot, \bar{x})$ respectively, i.e.,

$$
p=-D_{x} c\left(x, c-\operatorname{Exp}_{x} p\right) \text { for } p \in M^{*}(x), \quad \bar{p}=-D_{\bar{x}} c\left(\bar{c}-\operatorname{Exp}_{\bar{x}} \bar{p}, \bar{x}\right) \text { for } \bar{p} \in \bar{M}^{*}(\bar{x}) .
$$

Given a set $X$, we denote by $\operatorname{cl}(X)$ its closure. Define the subdifferential of a semiconvex funciton $\alpha: M \rightarrow \mathbb{R}$ at $x \in M$ by

$$
\partial \alpha(x):=\left\{p \in T_{x}^{*} M \mid \alpha\left(\exp _{x} v\right)-\alpha(x) \geq\langle p, v\rangle+o\left(|v|_{x}\right) \text { as } v \rightarrow 0 \text { in } T_{x} M\right\}
$$

(This is non-empty at every point.) Here $\langle\cdot, \cdot\rangle$ denotes the paring of covectors and vectors. 
Assumption 2.2. For each $(x, \bar{x}) \in M \times \bar{M}$ the map $c$-Exp $\operatorname{Ex}_{x}($ resp. $\bar{c}$-Exp $\bar{x})$ extends to a smooth map from $\mathrm{cl}\left(M^{*}(x)\right)$ (resp. $\left.\operatorname{cl}\left(\bar{M}^{*}(\bar{x})\right)\right)$ onto $\bar{M}$ (resp. $\left.M\right)$. If we abuse notation and use $c-\operatorname{Exp}_{x}, \bar{c}-\operatorname{Exp}_{\bar{x}}$ also for these extensions, then they satisfy

$$
\begin{array}{ll}
c-\operatorname{Exp}_{x} p=c-\operatorname{Exp}_{x}\left(\partial_{x}\left(-c\left(x, c-\operatorname{Exp}_{x} p\right)\right)\right), & \forall p \in \operatorname{cl}\left(M^{*}(x)\right) ; \\
\bar{c}-\operatorname{Exp}_{\bar{x}} \bar{p}=\bar{c}-\operatorname{Exp}_{\bar{x}}\left(\partial_{\bar{x}}\left(-c\left(c-\operatorname{Exp}_{\bar{x}} \bar{p}, \bar{x}\right)\right)\right), \quad \forall \bar{p} \in \operatorname{cl}\left(\bar{M}^{*}(\bar{x})\right) .
\end{array}
$$

Here, $\partial_{x}, \partial_{\bar{x}}$ denote the subdifferentials with respect to the variables $x$, $\bar{x}$, respectively.

Note that the above assumptions hold for instance when $M=\bar{M}$ and $c=\operatorname{dist}^{2} / 2$ (so that $c$-Exp $\operatorname{Ex}_{x}$ coincides with the Riemannian exponential map $\exp _{x}$ ). However, the following three assumptions are much more restrictive, and not true for $c=\mathrm{dist}^{2} / 2$ in general [MTW, L1, KM1, LV]. They are all crucial in this paper.

Assumption 2.3 (convexity of domains of $c$-exponentials). For each $(x, \bar{x}) \in M \times \bar{M}$ the domains $M^{*}(x), \bar{M}^{*}(\bar{x})$ are convex.

As shown in [FRV], the above assumption is necessary for continuity of optimal transport maps when the cost function is given by the squared distance.

A $c$-segment $\{\bar{x}(t)\}_{0 \leq t \leq 1}$ with respect to $x$ is the $c$-exponential image of a line segment in $\operatorname{cl}\left(M^{*}(x)\right)$, i.e.,

$$
\bar{x}(t):=c-\operatorname{Exp}_{x}\left((1-t) p_{0}+t p_{1}\right) \quad \text { for some } p_{0}, p_{1} \in T_{x}^{*} M .
$$

Define similarly a $\bar{c}$-segment $\{x(t)\}_{0 \leq t \leq 1}$ with respect to $\bar{x}$. The notions of $c$ - and $\bar{c}$ segments, due to Ma, Trudinger and Wang, induce a natural extension of the notion of convexity on sets in $M, \bar{M}$, called c-convexity in [MTW]. Let $U \subset M$ and $\bar{x} \in \bar{M}$. The set $U$ is said to be $\bar{c}$-convex with respect to $\bar{x}$ if any two points in $U$ are connected by a $\bar{c}$-segment with respect to $\bar{x}$ entirely contained inside $U$. Similarly we define $c$-convex sets in $\bar{M}$. It is helpful to notice that $c, \bar{c}$-convex sets (with respect to $x, \bar{x}$, respectively) are images of convex sets under $c$-Exp $\operatorname{Ex}_{x}, \bar{c}$-Exp $\bar{x}$, respectively.

Regarding $c, \bar{c}$-segments, here comes a key assumption in this paper:

Assumption 2.4 (convex DASM). For every $(x, \bar{x}) \in M \times \bar{M}$, let $\{\bar{x}(t)\}_{0 \leq t \leq 1},\{x(t)\}_{0 \leq t \leq 1}$ be $c, \bar{c}$-segments with respect to $x, \bar{x}$, respectively. Define the functions

$$
m_{t}(\cdot):=-c(\cdot, \bar{x}(t))+c(x, \bar{x}(t)), \quad \bar{m}_{t}(\cdot):=-c(x(t), \cdot)+c(x(t), \cdot), \quad 0 \leq t \leq 1 .
$$

Then

$$
m_{t} \leq(1-t) m_{0}+t m_{1}, \quad \bar{m}_{t} \leq(1-t) \bar{m}_{0}+t \bar{m}_{1}, \quad 0 \leq t \leq 1 .
$$

When, instead of (2.1), only $m_{t} \leq \max \left[m_{0}, m_{1}\right]$ and $\bar{m}_{t} \leq \max \left[\bar{m}_{0}, \bar{m}_{1}\right]$ are required, this property played a key role in the work of Loeper [L1]. In [KM1] we called it Loeper's maximum principle (DASM), the acronym (DASM) standing for "Double Mountain Above Sliding Mountain", a mnemonic which describes how the graphs of the functions $m_{t}, \bar{m}_{t}$ behave as $t$ is varied. For convenience we use this acronym in various places in the present paper. The stronger property (convex DASM) was proved in [KM2] to be a consequence of the so-called non-negative cross-curvature condition on the cost $c$.

We will also need a strict version of Loeper's maximum principle (DASM): 
Assumption 2.5 $\left(\mathbf{D A S M}^{+}\right)$. With the same notation as in Assumption 2.4,

$$
m_{t}(y) \leq \max \left[m_{0}(y), m_{1}(y)\right] \quad \forall y \in M, \quad \bar{m}_{t}(\bar{y}) \leq \max \left[\bar{m}_{0}(\bar{y}), \bar{m}_{1}(\bar{y})\right] \quad \forall \bar{y} \in \bar{M} .
$$

Moreover, when the $\bar{c}$-(resp. $c$-) segment in the definition of $m_{t}\left(\right.$ resp. $\left.\bar{m}_{t}\right)$ is non-constant, equality holds if and only if $y=x($ resp. $\bar{y}=\bar{x})$.

Assumptions 2.4 and 2.5 correspond to a "global" version of the non-negative cross curvature assumption and of the (A3) condition on the cost function $c$, respectively: see [KM1] and [MTW] for the definition of non-negative cross curvature and (A3), respectively. Although the equivalence between (convex DASM) and non-negative cross curvature (resp. $\left(\mathbf{D A S M}^{+}\right)$and (A3)) is not known in general, it holds true for the squared distance cost function on a Riemannian manifold, as shown in [FV, FRV]. Moreover, Loeper's maximum principle (DASM) is a necessary condition for regularity: this is originally shown [L1] on domains in $\mathbb{R}^{n}$ and later extended to the manifold case [FRV].

Given two functions $\phi: M \rightarrow \mathbb{R}$ and $\bar{\phi}: \bar{M} \rightarrow \mathbb{R}$, we say that they are $c$-convex and dual with respect to each other if

$$
\begin{aligned}
& \phi(x)=\sup _{\bar{x} \in \bar{M}}\{-c(x, \bar{x})-\bar{\phi}(\bar{x})\}, \\
& \bar{\phi}(\bar{x})=\sup _{x \in M}\{-c(x, \bar{x})-\phi(x)\}=\sup _{x \in M}\{-\bar{c}(\bar{x}, x)-\phi(x)\} .
\end{aligned}
$$

Since by assumption $c$ is semiconcave, both functions above are semiconvex (see for instance [FF, Appendix A]). This implies in particular that their subdifferentials $\partial \phi(x)$, $\partial \bar{\phi}(\bar{x})$ are non-empty at every point.

We define the $c$-subdifferential $\partial^{c} \phi$ at a point $x$ as follows:

$$
\partial^{c} \phi(x):=\{\bar{x} \in \bar{M} \mid \phi(y)-\phi(x) \geq-c(y, \bar{x})+c(x, \bar{x}), \forall y \in M\} .
$$

Analogously, we define $\partial^{\bar{c}} \bar{\phi}$ at every point $\bar{x}$. (Recall that $\bar{c}$ denotes the function defined as $\bar{c}(\bar{x}, x):=c(x, \bar{x})$.) The following well-known reciprocity holds:

Lemma 2.6 (Reciprocity). For c-convex functions $\phi, \bar{\phi}$ dual to each other as in (2.2),

$$
\bar{x} \in \partial^{c} \phi(x) \Leftrightarrow \phi(x)+\bar{\phi}(\bar{x})=-c(x, \bar{x}) \Leftrightarrow x \in \partial^{\bar{c}} \bar{\phi}(\bar{x}) .
$$

Proof. Suppose $\bar{x} \in \partial^{c} \phi(x)$. Then, by rearranging the inequality in (2.3) we get

$$
-\phi(x) \geq c(x, \bar{x})+\sup _{y \in M}\{-c(y, \bar{x})-\phi(y)\},
$$

and the supremum on the right hand side is exactly $\bar{\phi}(\bar{x})$. On the other hand, from the definition of $\phi$ and $\bar{\phi}$ we have

$$
\bar{\phi}(\bar{y})+c(x, \bar{y}) \geq-\phi(x) \quad \forall \bar{y} \in \bar{M},
$$

so that combining these two inequalities leads to $\phi(x)+\bar{\phi}(\bar{x})=-c(x, \bar{x})$, and $x \in$ $\partial^{\bar{c}} \bar{\phi}(\bar{x})$. The opposite implication follows by symmetry.

Loeper [L1] deduced the following fundamental relation to be a consequence of his maximum principle (DASM). 
Lemma 2.7 (Loeper's maximum principle (DASM)). Let Assumptions 2.1-2.3 hold. Suppose Loeper's maximum principle (DASM) holds. Let $\phi, \bar{\phi}$ be c-convex functions dual to each other as in (2.2). Then for all $x \in M, \bar{x} \in \bar{M}$,

$$
c-\operatorname{Exp}_{x}(\partial \phi(x))=\partial^{c} \phi(x), \quad \bar{c}-\operatorname{Exp}_{\bar{x}}(\partial \bar{\phi}(\bar{x}))=\partial^{\bar{c}} \bar{\phi}(\bar{x}) .
$$

Proof. The inclusions $c$-Exp $\operatorname{Ex}_{x}(\partial \phi(x)) \subset \partial^{c} \phi(x), \bar{c}-\operatorname{Exp}_{\bar{x}}(\partial \bar{\phi}(\bar{x})) \subset \partial^{\bar{c}} \bar{\phi}(\bar{x})$ follow from the convexity of $\partial \phi(x)$ and the definition of Loeper's maximum principle (DASM). The other inclusions hold in general without Loeper's maximum principle. Details can be found in [L1].

In the following, we refer to the conclusion of this lemma also as Loeper's maximum principle (DASM).

For a set $\Omega \subset M$, the image $\partial^{c} \phi(\Omega)$ is defined as

$$
\partial^{c} \phi(\Omega):=\bigcup_{x \in \Omega} \partial^{c} \phi(x) .
$$

For a $c$-convex function $\phi$ and an open set $U \subset M$ with $x_{0} \in U$, we define

$$
\left[\partial^{c} \phi(U)\right]_{x_{0}}:=\left\{\bar{x} \in \bar{M} \mid \phi(x)-\phi\left(x_{0}\right) \geq-c(x, \bar{x})+c\left(x_{0}, \bar{x}\right) \text { for all } x \in \partial U\right\} .
$$

Trivially, $\partial^{c} \phi\left(x_{0}\right) \subset\left[\partial^{c} \phi(U)\right]_{x_{0}}$. This definition is justified by the following lemma, which is also very useful in later discussions.

Lemma 2.8. Let Assumptions 2.1-2.3 hold. Suppose Loeper's maximum principle (DASM) holds. Let $\phi$ be a c-convex function on $M$. Let $U \subset M$ be an open set, and let $x_{0} \in U$. Then:

(1) $\left[\partial^{c} \phi(U)\right]_{x_{0}}$ is c-convex with respect to $x_{0}$.

(2) $\left[\partial^{c} \phi(U)\right]_{x_{0}} \subset \partial^{c} \phi(U)$.

(3) If $U \rightarrow\left\{x_{0}\right\}$, then both $\partial^{c} \phi(U),\left[\partial^{c} \phi(U)\right]_{x_{0}} \rightarrow \partial^{c} \phi\left(x_{0}\right)$.

Proof. Assertion (1) follows directly from the definitions of Loeper's maximum principle (DASM) and of the set $\left[\partial^{c} \phi(U)\right]_{x_{0}}$.

To prove (2), fix $\bar{x} \in\left[\partial^{c} \phi(U)\right]_{x_{0}}$, and move first the graph of the function $-c(\cdot, \bar{x})$ down so that it lies below $\phi$ inside $U$, and then lift it up until it touches the graph of $\phi$ inside $\operatorname{cl}(U)$. Thanks to the assumption $\bar{x} \in\left[\partial^{c} \phi(U)\right]_{x_{0}}$ there exists at least one touching point $x^{\prime}$ which belongs to $U$ (indeed, if there is a touching point on $\partial U$, then $x_{0}$ is another touching point), and Lemma 2.7 ensures that $\bar{x} \in \partial^{c} \phi\left(x^{\prime}\right)$.

For (3), the convergence $\partial^{c} \phi(U) \rightarrow \partial^{c} \phi\left(x_{0}\right)$ follows by continuity, and $\left[\partial^{c} \phi(U)\right]_{x_{0}}$ $\rightarrow \partial^{c} \phi\left(x_{0}\right)$ then comes from (2).

For $\bar{x} \in \bar{M}$, let $S(\bar{x})$ be the contact set

$$
S(\bar{x}):=\left\{x \in M \mid \bar{x} \in \partial^{c} \phi(x)\right\}=\partial^{\bar{c}} \bar{\phi}(\bar{x}) .
$$

(The last identity follows from reciprocity, see Lemma 2.6.) For any $x_{0} \in S(\bar{x})$ one can write

$$
S(\bar{x})=\left\{x \in M \mid \phi(x)-\phi\left(x_{0}\right)=-c(x, \bar{x})+c\left(x_{0}, \bar{x}\right)\right\} .
$$


A set $Z$ in $M$ is called a $c$-section of $\phi$ with respect to $\bar{x}$ if there is $\lambda_{\bar{x}} \in \mathbb{R}$ such that

$$
Z:=\left\{z \in M \mid \phi(z) \leq-c(z, \bar{x})+\lambda_{\bar{x}}\right\} .
$$

The following simple observation is very useful for studying regularity of $c$-convex functions. It was originally made (implicitly) in [FKM1] and independently by Liu [Li].

Lemma 2.9 (c-convex $c$-sections). Let Assumptions 2.1-2.3 hold. Suppose Loeper's maximum principle (DASM) holds. Let $\phi$ be a c-convex function on $M$, and fix $\bar{x} \in \bar{M}$. Every $c$-section $Z$ of $\phi$ with respect to $\bar{x}$ is $\bar{c}$-convex with respect to $\bar{x}$.

Proof. This follows from the definition of $c$-convex functions and of Loeper's maximum principle (DASM).

Given Borel sets $V \subset M$ and $\bar{V} \subset \bar{M}$, we denote by $|V|$ and $|\bar{V}|$ their volumes (computed with respect to the given Riemannian metric on $M$ and $\bar{M}$, respectively). The following is our last assumption. As we already remarked in the introduction, it is satisfied whenever $\phi$ is the potential associated to an optimal transport map and the densities are both bounded away from zero and infinity.

Assumption 2.10 (bounds on $c$-Monge-Ampère measure of $\phi$ ). There exists $\lambda>0$ such that

$$
\lambda|\Omega| \leq\left|\partial^{c} \phi(\Omega)\right| \leq \frac{1}{\lambda}|\Omega| \quad \text { for all Borel sets } \Omega \subset M .
$$

We sometimes abbreviate this condition on $\phi$ simply by writing $\left|\partial^{c} \phi\right| \in[\lambda, 1 / \lambda]$.

\section{Preliminary results}

In this section, we list some preliminary results we require later. The first subsection deals with general convex sets and the second subsection considers the properties of the cost function under suitable assumptions.

\subsection{Convex sets}

We first list two properties of convex sets that will be useful later.

Lemma 3.1 (John's lemma [J]). For a compact convex set $S \subset \mathbb{R}^{n}$, there exists an affine transformation $L: \mathbb{R}^{n} \rightarrow \mathbb{R}^{n}$ such that $B_{1} \subset L^{-1}(S) \subset B_{n}$. Here, $B_{1}$ and $B_{n}$ denote the balls of radius 1 and $n$, respectively, centered at 0 .

Lemma 3.2 ([FKM1, Lemma 6.11]). Let $S$ be a convex set in $\mathbb{R}^{n}=\mathbb{R}^{n^{\prime}} \times \mathbb{R}^{n^{\prime \prime}}$, and denote by $\pi^{\prime}, \pi^{\prime \prime}$ the canonical projections onto $\mathbb{R}^{n^{\prime}}$ and $\mathbb{R}^{n^{\prime \prime}}$, respectively. Let $S^{\prime}$ be a slice orthogonal to the second component, that is,

$$
S^{\prime}=\left(\pi^{\prime \prime}\right)^{-1}\left(\bar{x}^{\prime \prime}\right) \cap S \quad \text { for some } \bar{x}^{\prime \prime} \in \pi^{\prime \prime}(S) .
$$

Then there exists a constant $C(n)$, depending only on $n=n^{\prime}+n^{\prime \prime}$, such that

$$
C(n)|S| \geq \mathscr{H}^{n^{\prime}}\left(S^{\prime}\right) \mathscr{H}^{n^{\prime \prime}}\left(\pi^{\prime \prime}(S)\right),
$$

where $\mathscr{H}^{d}$ denotes the d-dimensional Hausdorff measure. 
The following lemma is important in the last step (Section 6.6) of the proof of the main theorem.

Lemma 3.3. Let $X=X^{1} \times \cdots \times X^{k}$, with $X^{i}=\mathbb{R}^{n_{i}}, i=1, \ldots, k$, and write a point $x \in X$ as $x=\left(x^{1}, \ldots, x^{k}\right), x^{i} \in X^{i}$. For each $i=1, \ldots, k$, let $U^{i}$ be a subset of $X^{i}$, and let $s_{i}=\left(s_{i}^{1}, \ldots, s_{i}^{k}\right) \in X$ with $s_{i}^{i} \in U^{i}$. Define $S_{i} \subset X$ as

$$
S_{i}:=\left\{s_{i}^{1}\right\} \times \cdots \times\left\{s_{i}^{i-1}\right\} \times U_{i} \times\left\{s_{i}^{i+1}\right\} \times \cdots \times\left\{s_{i}^{k}\right\},
$$

and consider the convex hull $\operatorname{co}\left(S_{1}, \ldots, S_{k}\right)$ of the sets $S_{1}, \ldots, S_{k}$. Then there exists a constant $C(n, k)$, depending only on $n:=n_{1}+\cdots+n_{k}$ and $k$, such that

$$
C(n, k)\left|\operatorname{co}\left(S_{1}, \ldots, S_{k}\right)\right| \geq \prod_{i=1}^{k} \mathscr{H}^{n_{i}}\left(S^{i}\right) .
$$

Proof. First consider the barycenter $b$ of the set $\left\{s_{1}, \ldots, s_{k}\right\}$, that is,

$$
b:=\frac{1}{k}\left(s_{1}+\cdots+s_{k}\right) .
$$

We will construct sets $S_{i}^{b}$ each of which contains $b$ and has Hausdorff measure comparable with $S_{i}$. In addition, these sets are mutually orthogonal. We will finish the proof by considering the volume of the convex hull of these sets $S_{1}^{b}, \ldots, S_{k}^{b}$.

For each $i$, let $b_{i}$ be the barycenter of the set $\left\{s_{1}, \ldots, s_{k}\right\} \backslash\left\{s_{i}\right\}$, i.e.,

$$
b_{i}:=\frac{1}{k-1}\left(s_{1}+\cdots+s_{i-1}+s_{i+1}+\cdots+s_{k}\right) .
$$

Consider the cone $\operatorname{co}\left(b_{i}, S_{i}\right) \subset \operatorname{co}\left(S_{1}, \ldots, S_{k}\right)$ and let

$$
S_{i}^{b}:=\operatorname{co}\left(b_{i}, S_{i}\right) \cap\left\{x \in X \mid x^{j}=b^{j} \text { for } j \neq i\right\} .
$$

Note that $b \in S_{i}^{b}$ and these sets $S_{1}^{b}, \ldots, S_{k}^{b}$ are mutually orthogonal, in the sense that, for each $x \in S_{i}^{b}$ and $y \in S_{j}^{b}$ with $i \neq j$, we have $(x-b) \cdot(y-b)=0$. Now, consider the convex hull $\operatorname{co}\left(S_{1}^{b}, \ldots, S_{k}^{b}\right) \subset \operatorname{co}\left(S^{1}, \ldots, S^{k}\right)$. The previous orthogonality implies

$$
C(n)\left|\operatorname{co}\left(S_{1}^{b}, \ldots, S_{k}^{b}\right)\right| \geq \prod_{i=1}^{k} \mathscr{H}^{n_{i}}\left(S_{i}^{b}\right)
$$

for some constant $C(n)$ depending only on $n_{1}+\cdots+n_{k}$. (This inequality is obtained for instance by iteratively applying Lemma 3.2.) To conclude the proof simply observe that $b=\frac{1}{k} s_{i}+\frac{k-1}{k} b_{i}$, and so

$$
\mathscr{H}^{n_{i}}\left(S_{i}^{b}\right) \geq \frac{1}{k^{n_{i}}} \mathscr{H}^{n_{i}}\left(S_{i}\right)
$$

\subsection{Coordinate change}

In this subsection we briefly recall the coordinate change introduced in [FKM1, Section 4] that transforms $c$-convex functions into convex functions under the condition (convex DASM), referring to [FKM1, Section 4] for more details. Throughout this subsection we let Assumptions 2.1-2.3 hold. 
Let $\bar{y}_{0}$ be an arbitrary point in $\bar{M}$. Then the map $x \in M\left(\bar{y}_{0}\right) \mapsto q \in T_{\bar{y}_{0}}^{*} \bar{M}$ given by $q(x)=-D_{\bar{x}} c\left(x, \bar{y}_{0}\right)$ is an embedding thanks to Assumption 2.1. Recall that $\bar{M}^{*}\left(\bar{y}_{0}\right) \subset$ $T_{\bar{y}_{0}}^{*} \bar{M}$ denotes the image of this map, that this map is by definition the inverse $c$-exponential map $\left(c-\operatorname{Exp}_{\bar{y}_{0}}\right)^{-1}$, and the $c$-exponential map is a diffeomorphism up to the boundary of $\bar{M}^{*}\left(\bar{y}_{0}\right)$ (see Assumption 2.2). Denote

$$
\tilde{c}(q, \bar{x}):=c(x(q), \bar{x})-c\left(x(q), \bar{y}_{0}\right) .
$$

Then the $c$-convex function $\phi$ is transformed to a $\tilde{c}$-convex function $\varphi$ defined as

$$
\varphi(q):=\phi(x(q))+c\left(x(q), \bar{y}_{0}\right) .
$$

If Loeper's maximum principle (DASM) holds, then Lemma 2.9 shows that $\tilde{c}$-sections of $\varphi$ are convex. This property was observed independently by Liu [Li], who used it to derive an optimal Hölder exponent for optimal maps under the strict condition (A3) on the cost, sharpening the Hölder continuity result of Loeper [L1]. Furthermore, if (convex DASM) holds then $-\tilde{c}(q, \bar{x})$ is convex in $q$ for any $\bar{x} \in \bar{M}$, which then implies convexity of $\varphi$ in $q$ (see [FKM1, Theorem 4.3] for more details). One can easily check that $\bar{c}$ segments with respect to $\bar{x}$ are transformed via this coordinate change to $\tilde{\bar{c}}$-segments with respect to $\bar{x}$, and $c$-segments with respect to $x(q)$ are transformed to $\tilde{c}$-segments with respect to $q$. Therefore, Loeper's maximum principle (DASM) or (convex DASM) for $c$ implies the same for $\tilde{c}$.

3.2.1. Relation between cotangent vectors in two different coordinates. Here we give an explicit relation between covectors in the new coordinate variable $q$ (as introduced above) and the original coordinate variable $x$. Fix arbitrary $\bar{y}_{0} \in M, x_{0} \in M\left(\bar{y}_{0}\right)$, and let $q_{0}=-D_{\bar{x}} c\left(x_{0}, \bar{y}_{0}\right) \in T_{\bar{y}_{0}}^{*} \bar{M}$. For each $\bar{z} \in \bar{M}\left(x_{0}\right)$, consider the maps

$$
\begin{aligned}
& \bar{z} \mapsto \eta(\bar{z}):=-D_{x} c\left(x_{0}, \bar{z}\right) \in T_{x_{0}}^{*} M, \\
& \bar{z} \mapsto p(\bar{z}):=-D_{q} \tilde{c}\left(q_{0}, \bar{z}\right) \in T_{q_{0}}^{*}\left(T_{\bar{y}_{0}}^{*} \bar{M}\right) .
\end{aligned}
$$

where $\tilde{c}(q, \bar{x}):=c(x(q), \bar{x})-c\left(x(q), \bar{y}_{0}\right)$ and the variables $x$ and $q$ are related as $q(x)=$ $-D_{\bar{x}} c\left(x, \bar{y}_{0}\right)$. Denote by $M^{*}\left(x_{0}\right), \tilde{M}^{*}\left(q_{0}\right)$ the embedding of $\bar{M}\left(x_{0}\right)$ under the mappings $\bar{z} \mapsto \eta(\bar{z}), \bar{z} \mapsto p(\bar{z})$, respectively. These sets are related by an affine map as we see in the following lemma. In particular, from Assumption 2.3 both sets are convex in $T_{x_{0}}^{*} M$, $T_{q_{0}}^{*}\left(T_{\bar{y}_{0}}^{*} M\right)$, respectively.

Lemma 3.4. Let Assumptions 2.1 and 2.2 hold. Let $\eta(p): \tilde{M}^{*}\left(q_{0}\right) \rightarrow M^{*}\left(x_{0}\right)$ associate $p(\bar{z})$ to $\eta(\bar{z})$ as in (3.1), and let $\eta_{0}=-D_{x} c\left(x_{0}, \bar{y}_{0}\right) \in M^{*}\left(x_{0}\right) \subset T_{x_{0}}^{*}$ M. Fix local coordinates. Then for all $p \in \tilde{M}^{*}\left(q_{0}\right), \eta(p)=\left(\eta(p)^{1}, \ldots, \eta(p)^{n}\right)$ is given as

$$
\eta(p)^{i}=p^{j}\left(-D_{x^{i}} D_{\bar{x}^{j}} c\left(x_{0}, \bar{y}_{0}\right)\right)+\eta_{0}^{i} .
$$

This formula allows the affine function $p \mapsto \eta(p)$ to be extended to a global map $\eta$ : $T_{q_{0}}^{*}\left(T_{\bar{y}_{0}}^{*} \bar{M}\right) \rightarrow T_{x_{0}}^{*} M$. 
Proof. Observe that

$$
\begin{aligned}
\eta^{i} & =-D_{x^{i}} c\left(x_{0}, \bar{z}\right)=\left.D_{x^{i}}\right|_{x=x_{0}}\left[-c(x, \bar{z})+c\left(x, \bar{y}_{0}\right)-c\left(x, \bar{y}_{0}\right)\right] \\
& =-\left.D_{q^{j}}\right|_{q=q_{0}}\left[c(x(q), \bar{z})-c\left(x(q), \bar{y}_{0}\right)\right]\left(\left.D_{x^{i}}\right|_{x=x_{0}} q^{j}\right)+\eta_{0}^{i}=p^{j}\left(\left.D_{x^{i}}\right|_{x=x_{0}} q^{j}\right)+\eta_{0}^{i} .
\end{aligned}
$$

From the relation $q^{j}=-D_{\bar{x}^{j}} c\left(x(q), \bar{y}_{0}\right)$ we see that

$$
\left.D_{x^{i}}\right|_{x=x_{0}} q=-D_{x^{i}} D_{\bar{x}^{j}} c\left(x_{0}, \bar{y}_{0}\right),
$$

and the assertion follows.

3.2.2. An estimate on the first derivatives of $c$. In Section 6.5 we will use the following simple estimate.

Lemma 3.5 ([FKM1, Lemma 6.3]). Given convex sets $\Omega, \Lambda \subset \mathbb{R}^{n}$, assume that the function $(q, y) \in \Omega \times \Lambda \mapsto c(q, y) \in \mathbb{R}$ is smooth. Then for all $q, \tilde{q} \in \Omega$ and $y \in \Lambda$,

$$
\left|-D_{q} c(q, y)+D_{q} c(\tilde{q}, y)\right| \leq C|q-\tilde{q}|\left|D_{q} c(\tilde{q}, y)\right|,
$$

where the constant $C$ depends only on $\|c\|_{C^{3}(\Omega \times \Lambda)}$ and $\left\|\left(D_{q y}^{2} c\right)^{-1}\right\|_{L^{\infty}(\Omega \times \Lambda)}$.

\section{An Alexandrov estimate: lower bound}

In this section we show a key Alexandrov type estimate (4.1) which bounds from below the "oscillation" of $\phi$ inside a $c$-section by the measure of the section. (An estimate that compares the oscillation of the function with the measure of the section is said to be of Alexandrov type.) This result is of its own interest, especially because it is proven under rather general assumptions, and does not rely on the special structure of products of spheres. In later sections, a companion inequality showing the upper bound will be obtained for a special choice of a $c$-section in the particular case of products of spheres (see Theorem 6.4).

Lemma 4.1 (Alexandrov lower bound). Let $M, \bar{M}$ be complete $n$-dimensional Riemannian manifolds. Suppose the cost c $: M \times \bar{M} \rightarrow \mathbb{R}$ satisfies Assumptions 2.1-2.4. Let $\phi$ be a c-convex function on $M$ and assume $0<\lambda \leq\left|\partial^{c} \phi\right|$ for a fixed $\lambda \in \mathbb{R}$. Fix $\left(x_{0}, \bar{x}_{0}\right) \in M \times \bar{M}$ such that $\bar{x}_{0} \in \partial^{c} \phi\left(x_{0}\right)$, and for $\bar{h}>0$ consider the c-section $Z_{h}$ defined as

$$
Z_{h}:=\left\{x \in M \mid \phi(x)-\phi\left(x_{0}\right) \leq-c\left(x, \bar{x}_{0}\right)+c\left(x_{0}, \bar{x}_{0}\right)+h\right\} .
$$

Assume that $-c\left(\cdot, \bar{x}_{0}\right)$ is smooth on $Z_{h}$, so that the function $\bar{c}-\operatorname{Exp}_{\bar{x}_{0}}^{-1}$ is defined and smooth on $Z_{h}$, or equivalently $Z_{h} \subset M\left(\bar{x}_{0}\right)$. Then

$$
\lambda\left|Z_{h}\right|^{2} \leq C(n)\left[\frac{\max _{x \in Z_{h}}\left|\operatorname{det}\left(-D_{x} D_{\bar{x}} c\left(x, \bar{x}_{0}\right)\right)\right|}{\min _{x \in Z_{h}}\left|\operatorname{det}\left(-D_{x} D_{\bar{x}} c\left(x, \bar{x}_{0}\right)\right)\right|}\right]^{2}\left[\sup _{x \in Z_{h}} \sup _{p^{\prime} \in M^{*}(x)}\left|d c-\operatorname{Exp}_{x}\right|_{p=p^{\prime}} \mid\right] h^{n}
$$

with the constant $C(n)=(4 n)^{n}\left|B_{1}\right|^{2}$, where $\left|B_{1}\right|$ denotes the measure of the unit ball in $\mathbb{R}^{n}$. 
Remark 4.2. In the statement of Lemma 4.1 and its proof, it is important to notice that by the assumption that $-c\left(\cdot, \bar{x}_{0}\right)$ is smooth on $\mathrm{cl} Z_{h}$ and by Assumptions 2.1 and 2.2, the derivatives of $\bar{c}$-Exp $\bar{x}_{0}$ and its inverse (on $Z_{h}$ ), i.e., $-D_{x} D_{\bar{x}} c\left(x, \bar{x}_{0}\right), x \in Z_{h}$, are all nonsingular.

Remark 4.3. For $c=\operatorname{dist}^{2} / 2$, Loeper's maximum principle (DASM) (and so also (convex DASM)) implies that $M=\bar{M}$ has non-negative sectional curvature (see [L1]). Therefore in this case $c$ - $\operatorname{Exp}_{y}$ is a contraction, that is,

$$
\sup _{p^{\prime} \in M^{*}(x)}\left|d c-\operatorname{Exp}_{x}\right|_{p=p^{\prime}} \mid \leq 1
$$

We do not know if this contraction property holds for general non-negatively cross-curved cost functions.

Remark 4.4. As in [FKM1], for our main results we will later follow the strategy developed in [Ca1] by using renormalization techniques, but only after a suitable change of coordinates. The main feature of our Alexandrov estimate with respect to the ones in [Ca1, FKM1] is that the only "possibly bad" dependence on the cost function comes from the term $\max _{x \in Z_{h}}\left|\operatorname{det}\left(-D_{x} D_{\bar{x}} c\left(x, \bar{x}_{0}\right)\right)\right| / \min _{x \in Z_{h}}\left|\operatorname{det}\left(-D_{x} D_{\bar{x}} c\left(x, \bar{x}_{0}\right)\right)\right|$, which can be made as close to 1 as desired, provided one can ensure that the section $Z_{h}$ converges to a point as $h \rightarrow 0$. This will play a crucial role in the proof of Theorem 5.1, as it will allow us to apply this estimate near points arbitrarily close to the cut-locus.

Proof. For globally smooth cost functions (on the products of two bounded domains) a similar result was proved in [FKM1, Theorem 6.4]. In the present case where the cost function has singularities, the previous proof does not work any more and we require the following subtle argument.

Consider the coordinate change $x \in Z_{h} \mapsto q=-D_{\bar{x}} c\left(x(q), \bar{x}_{0}\right) \in \bar{W}_{h} \subset \bar{M}^{*}\left(\bar{x}_{0}\right) \subset$ $T_{\bar{x}_{0}}^{*} \bar{M}$, i.e., $x=c$-Exp $\bar{x}_{0} q$ and $Z_{h}=c$-Exp $\operatorname{Ex}_{\bar{x}_{0}}\left(\bar{W}_{h}\right)$, and let

$$
m_{\bar{x}}(\cdot):=-c(\cdot, \bar{x})+c\left(\cdot, \bar{x}_{0}\right) .
$$

As explained in Section 3.2, in these new coordinates the functions

$$
q \mapsto m_{\bar{x}}(x(q)) \quad \text { and } \quad q \mapsto \varphi(q)=\phi(x(q))+c\left(x(q), \bar{x}_{0}\right)
$$

are convex. Moreover the set $\bar{W}_{h}$ is convex, as

$$
\bar{W}_{h}=\left\{q \in T_{\bar{x}_{0}}^{*} \bar{M} \mid \varphi(q)-\varphi\left(q_{0}\right) \leq h\right\},
$$

where $q_{0}$ is the point corresponding to $x_{0}$ in the new coordinates, i.e., $c$-Exp $\bar{x}_{\bar{x}_{0}} q_{0}=x_{0}$. It is also important to notice that $\bar{x}_{0} \in \partial^{c} \phi\left(x_{0}\right)$ implies $\varphi(q)-\varphi\left(q_{0}\right) \geq 0$. We now use Lemma 3.1 to find an affine map $A: T_{\bar{x}_{0}}^{*} \bar{M} \simeq \mathbb{R}^{n} \mapsto T_{\bar{x}_{0}}^{*} \bar{M} \simeq \mathbb{R}^{n}$ such that $A\left(\hat{W}_{h}\right)=\bar{W}_{h}$ with $B_{1} \subset \hat{W}_{h} \subset B_{n}$. Denote $q_{b}=A(0)$ and $x_{b}=c$ - $\operatorname{Exp}_{\bar{x}_{0}} q_{b}$. Define the renormalized function $\hat{\varphi}(\hat{q}):=\varphi(A \hat{q})$ for each $\hat{q} \in \hat{W}_{h}$, and denote $\frac{1}{2} \bar{W}_{h}:=A\left(\frac{1}{2} \hat{W}_{h}\right)$ and $\frac{1}{2} Z_{h}:=$ 
$\bar{c}$ - $\operatorname{Exp}_{\bar{x}_{0}}\left(\frac{1}{2} \bar{W}_{h}\right)$, where $\frac{1}{2} \hat{W}_{h}$ denotes the dilation of $\hat{W}_{h}$ by a factor $1 / 2$ with respect to the origin. This $1 / 2$-dilation (any factor in $(0,1)$ works) will be important in this proof.

Consider the reciprocal expression

$$
\partial^{c} \phi\left(\frac{1}{2} Z_{h}\right)=c-\operatorname{Exp}_{x_{b}}\left(-D_{x} c\left(x_{b}, \bar{x}_{0}\right)+\mathcal{V}\right)
$$

where

$$
\mathcal{V}:=\left\{D_{x} m_{\bar{x}}\left(x_{b}\right) \mid \bar{x} \in \partial^{c} \phi\left(\frac{1}{2} Z_{h}\right)\right\} \subset T_{x_{b}}^{*} M .
$$

Here $D_{x} m_{\bar{x}}$ denotes the differential when $m_{\bar{x}}$ is differentiable, otherwise it means an arbitrary covector in the subdifferential $\partial m_{\bar{x}}(x)$. Notice that $-D_{x} c\left(x_{b}, \bar{x}_{0}\right)+\mathcal{V} \subset \operatorname{cl}\left(M^{*}\left(x_{b}\right)\right)$, and thus

$$
\left|\partial^{c} \phi\left(\frac{1}{2} Z_{h}\right)\right| \leq\left(\sup _{p^{\prime} \in M^{*}\left(x_{b}\right)}\left|d c-\operatorname{Exp}_{x_{b}}\right|_{p=p^{\prime}} \mid\right)|\mathcal{V}| .
$$

Now, the left-hand side is bounded from below as

$$
\begin{aligned}
& \left.\left|\partial^{c} \phi\left(\frac{1}{2} Z_{h}\right)\right| \geq \lambda\left|\frac{1}{2} Z_{h}\right| \quad \text { (by the assumption }\left|\partial^{c} \phi\right| \geq \lambda\right) \\
& \geq \lambda\left[\min _{w \in W_{h}}\left|\operatorname{det}\left(d \bar{c}-\left.\operatorname{Exp}_{\bar{x}_{0}}\right|_{q=w}\right)\right|\right]\left(\frac{1}{2}\right)^{n}\left|W_{h}\right| \quad\left(\text { since } \frac{1}{2} Z_{h}=\bar{c}-\operatorname{Exp}_{\bar{x}_{0}}\left(\frac{1}{2} W_{h}\right)\right) \\
& \geq \lambda \frac{\min _{w \in W_{h}}\left|\operatorname{det}\left(d \bar{c}-\operatorname{Exp}_{\bar{x}_{0}} \mid q=w\right)\right|}{\max _{w \in W_{h}}\left|\operatorname{det}\left(d \bar{c}-\left.\operatorname{Exp}_{\bar{x}_{0}}\right|_{q=w}\right)\right|}\left(\frac{1}{2}\right)^{n}\left|Z_{h}\right| \\
& \geq \lambda \frac{\min _{x \in Z_{h}}\left|\operatorname{det}\left(-D_{x} D_{\bar{x}} c\left(x, \bar{x}_{0}\right)\right)\right|}{\max _{x \in Z_{h}}\left|\operatorname{det}\left(-D_{x} D_{\bar{x}} c\left(x, \bar{x}_{0}\right)\right)\right|}\left(\frac{1}{2}\right)^{n}\left|Z_{h}\right| \quad\left(\text { since } D_{\bar{x}} c\left(\cdot, \bar{x}_{0}\right)=\bar{c}-\operatorname{Exp}_{\bar{x}_{0}}^{-1}\right) .
\end{aligned}
$$

In the following we will bound $|\mathcal{V}|$ from above by

$$
\frac{\max _{x \in Z_{h}}\left|\operatorname{det}\left(-D_{x} D_{\bar{x}} c\left(x, \bar{x}_{0}\right)\right)\right|}{\min _{x \in Z_{h}}\left|\operatorname{det}\left(-D_{x} D_{\bar{x}} c\left(x, \bar{x}_{0}\right)\right)\right|} \frac{h^{n}}{\left|Z_{h}\right|},
$$

which will finish the proof; here the dilation $\frac{1}{2} Z_{h}$ plays a crucial role (see (4.5)). Fix $\bar{x} \in \partial^{c} \phi\left(\frac{1}{2} Z_{h}\right)$, and let $\hat{q}_{\bar{x}} \in \frac{1}{2} \hat{W}_{h}$ be such that $\bar{x} \in \partial^{\hat{c}} \hat{\varphi}\left(\hat{q}_{\bar{x}}\right)$. Here, $\hat{c}$ is the cost function modified accordingly with the coordinate changes:

$$
\hat{c}(\hat{q}, \bar{y}):=c\left(\bar{c}-\operatorname{Exp}_{\bar{x}_{0}}(A \hat{q}), \bar{y}\right)-c\left(\bar{c}-\operatorname{Exp}_{\bar{x}_{0}}(A \hat{q}), \bar{x}_{0}\right) .
$$

Consider the function

$$
\hat{m}_{\bar{x}}(\hat{q}):=m_{\bar{x}}\left(\bar{c}-\operatorname{Exp}_{\bar{x}_{0}}(A \hat{q})\right)=-\hat{c}(\hat{q}, \bar{x}) .
$$

Then

$$
\hat{m}_{\bar{x}}(\hat{q})-\hat{m}_{\bar{x}}\left(\hat{q}_{\bar{x}}\right)+\hat{\varphi}\left(\hat{q}_{\bar{x}}\right) \leq \hat{\varphi}(\hat{q}) \quad \text { for } \hat{q} \in \hat{W}_{h} .
$$

We observe that $\hat{m}_{\bar{x}}(\cdot)-\hat{m}_{\bar{x}}\left(\hat{q}_{\bar{x}}\right)$ is a convex function on $\hat{W}_{h}$ which vanishes at $\hat{q}_{\bar{x}} \in$ $\frac{1}{2} \hat{W}_{h}$, and $\hat{m}_{\bar{x}}(\cdot)-\hat{m}_{\bar{x}}\left(\hat{q}_{\bar{x}}\right) \leq h$ on $\partial \hat{W}_{h}$. Since $B_{1} \subset \hat{W}_{h} \subset B_{n}$ this easily implies that $\hat{m}_{\bar{x}}(0)-\hat{m}_{\bar{x}}\left(\hat{q}_{\bar{x}}\right) \geq-h$, which by convexity gives

$$
\left|D_{\hat{q}} \hat{m}_{\bar{x}}(0)\right| \leq 2 h .
$$


To get information on $D_{x} m_{\bar{x}}$, observe that from (4.4),

$$
\left(d \bar{c}-\left.\operatorname{Exp}_{\bar{x}_{0}}\right|_{q=q_{b}} A\right)^{*} D_{x} m_{\bar{x}}\left(x_{b}\right)=D_{\hat{q}} \hat{m}_{\bar{x}}(0),
$$

where $\left(d \bar{c}-\left.\operatorname{Exp}_{\bar{x}_{0}}\right|_{q=q_{b}} A\right)^{*}: T_{x_{b}}^{*} M \rightarrow T_{0}^{*}\left(T_{\bar{x}_{0}}^{*} \bar{M}\right)$ is the dual map of the derivative map $d \bar{c}-\operatorname{Exp}_{\bar{x}_{0}} A: T_{0}\left(T_{\bar{x}_{0}}^{*} \bar{M}\right) \rightarrow T_{x_{b}} M$. Here we abuse the notation and $A$ denotes both the affine map and its derivative. Moreover we use the canonical identification $T_{0}^{*}\left(T_{\bar{x}_{0}}^{*} \bar{M}\right) \approx$ $T_{0}\left(T_{\bar{x}_{0}}^{*} \bar{M}\right) \approx T_{\bar{x}_{0}}^{*} \bar{M}$. Hence (4.5) and (4.6) imply the key inclusion

$$
\mathcal{V} \subset\left(d c-\left.\operatorname{Exp}_{\bar{x}_{0}}\right|_{q=q_{b}} ^{*}\right)^{-1}\left(A^{*}\right)^{-1} B_{2 h}
$$

so that

$$
\begin{aligned}
& |\mathcal{V}| \leq\left|\operatorname{det}\left(d \bar{c}-\left.\operatorname{Exp}_{\bar{x}_{0}}\right|_{q=q_{b}} ^{*}\right)^{-1}\right|\left|\operatorname{det}\left(A^{*}\right)^{-1}\right|\left|B_{1}\right| 2^{n} h^{n} \\
& =\left|\operatorname{det}\left(d \bar{c}-\left.\operatorname{Exp}_{\bar{x}_{0}}\right|_{q=q_{0}}\right)\right|^{-1}|\operatorname{det} A|^{-1}\left|B_{1}\right| 2^{n} h^{n}
\end{aligned}
$$

(by the identification between vectors and covectors)

$$
\begin{aligned}
\leq & \left|\operatorname{det}\left(d \bar{c}-\left.\operatorname{Exp}_{\bar{x}_{0}}\right|_{q=q_{0}}\right)\right|^{-1}\left|B_{1}\right|^{2}(2 n)^{n} \frac{h^{n}}{\left|W_{h}\right|}\left(\text { since }\left|W_{h}\right|=|\operatorname{det} A|\left|\hat{W}_{h}\right| \leq|\operatorname{det} A|\left|B_{1}\right| n^{n}\right) \\
\leq & \frac{\max _{w \in W_{h}}\left|\operatorname{det}\left(d \bar{c}-\operatorname{Exp}_{\bar{x}_{0}} \mid q=w\right)\right|}{\left|\operatorname{det}\left(d \bar{c}-\left.\operatorname{Exp}_{\bar{x}_{0}}\right|_{q=q_{0}}\right)\right|}\left|B_{1}\right|^{2}(2 n)^{n} \frac{h^{n}}{\left|Z_{h}\right|} \\
& \left(\operatorname{since}\left|Z_{h}\right| \leq \max _{w \in W_{h}}\left|\operatorname{det}\left(d \bar{c}-\left.\operatorname{Exp}_{\bar{x}_{0}}\right|_{q=w}\right)\right|\left|W_{h}\right|\right) \\
\leq & \frac{\left.\max _{x \in Z_{h}}\left|\operatorname{det}\left(-D_{x} D_{\bar{x}} c\left(x, \bar{x}_{0}\right)\right)\right|\right)}{\min _{x \in Z_{h}}\left|\operatorname{det}\left(-D_{x} D_{\bar{x}} c\left(x, \bar{x}_{0}\right)\right)\right|}\left|B_{1}\right|^{2}(2 n)^{n} \frac{h^{n}}{\left|Z_{h}\right|} \quad\left(\text { since } D_{\bar{x}} c\left(\cdot, \bar{x}_{0}\right)=\bar{c}-\operatorname{Exp}_{\bar{x}_{0}}^{-1}\right)
\end{aligned}
$$

Together with (4.2) and (4.3), this concludes the proof.

\section{Stay-away property on multiple products of spheres}

From now on we restrict our attention to the case $M=\bar{M}=M^{1} \times \cdots \times M^{k}$, where for each $i=1, \ldots, k, M^{i}=\mathbb{S}_{r_{i}}^{n_{i}}$ is a round sphere of constant sectional curvature $r_{i}^{-2}$. Though $M=\bar{M}$, we sometimes keep the bar notation to emphasize the distinction between the source and the target domain of the transportation. Let $x=\left(x^{1}, \ldots, x^{k}\right)$ and $\bar{x}=\left(\bar{x}^{1}, \ldots, \bar{x}^{k}\right)$ denote points in $M^{1} \times \cdots \times M^{k}$, with $x^{i}, \bar{x}^{i} \in M^{i}, i=1, \ldots, k$. Assume that the transportation cost $c$ on $M$ is the tensor product of the $\operatorname{costs} c^{i}$ on each $M^{i}$, defined as

$$
c(x, \bar{x}):=\sum_{i=1}^{k} c^{i}\left(x^{i}, \bar{x}^{i}\right) .
$$

Assume moreover that each $c^{i}$ is of the form $f^{i}\left(\right.$ dist $\left._{i}\right)$ (dist $t_{i}$ being the distance on $\left.M^{i}\right)$ for some smooth strongly convex even function $f^{i}: \mathbb{R} \rightarrow \mathbb{R}$, normalized so that $f^{i}(0)=0$. (This normalization assumption can be made with no loss of generality, as one can always 
add an arbitrary constant to the cost function.) Moreover we suppose that each $c^{i}$ satisfies Assumptions 2.1-2.5 in Section 2. As shown in [KM2], under these assumptions the tensor product cost $c$ also satisfies Assumptions 2.1-2.4 (but not necessarily 2.5). The reader should have in mind that our model example is $c=\operatorname{dist}^{2} / 2$, which as shown in [KM2] satisfies all the assumptions above. However we prefer to give a proof of the result with general $f^{i}$ since this will not cost further effort in the proof, and we believe it may be of interest for future applications.

Let us observe that for any point $\bar{x}$ we have $M(\bar{x})=M^{1}\left(\bar{x}^{1}\right) \times \cdots \times M^{k}\left(\bar{x}^{k}\right)$ and $\bar{M}^{*}(\bar{x})=\bar{M}^{*}\left(\bar{x}^{1}\right) \times \cdots \times \bar{M}^{*}\left(\bar{x}^{k}\right)$. Moreover, since the distance squared function on a round sphere is smooth except for antipodal pairs, for each $x^{i} \in M^{i}$ we have $\operatorname{Cut}\left(x^{i}\right)=\left\{-x^{i}\right\}$, where $-x^{i}$ denotes the antipodal point of $x^{i}$. (We also write $-x=\left(-x^{1}, \ldots,-x^{k}\right)$.) This implies easily that $c$-Cut $(x)=\operatorname{Cut}(x)$, so that $M^{i}\left(x^{i}\right)=$ $M^{i} \backslash\left\{-x^{i}\right\}$ and $c$-Cut $(x)$ is a union of (totally geodesic) submanifolds, each of which is an embedding of a product $M^{i_{1}} \times \cdots \times M^{i_{l}}, l<k$.

The goal of the rest of the paper is to show a stay-away property of optimal transport maps on products of spheres:

Theorem 5.1 (Stay-away from cut-locus). Let $M=\bar{M}=M^{1} \times \cdots \times M^{k}$, where for each $i=1, \ldots, k, M^{i}=\mathbb{S}_{r_{i}}^{n_{i}}$ is a round sphere of constant sectional curvature $r_{i}^{-2}$. Let $c$ be the cost given in (5.1) with $c^{i}$ of the form $f^{i}\left(\right.$ dist $\left._{i}\right)$, where $f^{i}: \mathbb{R} \rightarrow \mathbb{R}$ are smooth strongly convex even functions such that $f^{i}(0)=0$. Assume further that each $c^{i}$ satisfies Assumptions 2.1-2.5, and let $\phi$ be a c-convex function satisfying Assumption 2.10. Then

$$
\partial^{c} \phi(x) \cap c-\operatorname{Cut}(x)=\emptyset \quad \forall x \in M .
$$

Equivalently, for every $\bar{x} \in \bar{M}$ the contact set $S(\bar{x})=\partial^{\bar{c}} \bar{\phi}(\bar{x})$ satisfies

$$
S(\bar{x}) \cap \bar{c}-\operatorname{Cut}(\bar{x})=\emptyset \text {. }
$$

Before sketching the proof of this result, let us first see its consequences:

Corollary 5.2 (Uniformly stay-away from cut-locus). Under the notation and assumptions as in Theorem 5.1, there exists a positive constant $C$ depending only on $\lambda$ (see Assumption 2.10) and $n_{i}, r_{i}, f^{i}$, for $i=1, \ldots, k$, such that

$$
\operatorname{dist}\left(\partial^{c} \phi(x), c-\operatorname{Cut}(x)\right) \geq C \quad \forall x \in M .
$$

where dist denotes the Riemannian distance of $M$.

Proof. The result follows by compactness. Indeed, suppose for a contradiction that there exists a sequence of $c$-convex functions $\phi_{l}$ satisfying Assumption 2.10, and $x_{l} \in M$, such that

$$
\operatorname{dist}\left(\partial^{c} \phi_{l}\left(x_{l}\right), c-\operatorname{Cut}\left(x_{l}\right)\right) \rightarrow 0 \quad \text { as } l \rightarrow \infty .
$$

Up to adding a constant, we can also assume that $\phi_{l}\left(x_{l}\right)=0$. Then, since $M$ is compact and the functions $\phi_{l}$ are uniformly semiconvex (and so uniformly Lipschitz), applying Arzelà-Ascoli's Theorem, up to a subsequence there exists a $c$-convex function $\phi_{\infty}$ and 
$x_{\infty} \in M$ such that $\phi_{l} \rightarrow \phi_{\infty}$ uniformly and $x_{l} \rightarrow x_{\infty}$. We now observe that also $\phi_{\infty}$ satisfies Assumption 2.10 (see for instance [FKM1, Lemma 3.1]). Moreover, by the definition of $c$-subdifferential we easily obtain

$$
y_{l} \in \partial^{c} \phi_{l}\left(x_{l}\right), y_{l} \rightarrow y_{\infty} \Rightarrow y_{\infty} \in \partial^{c} \phi_{\infty}\left(x_{\infty}\right) .
$$

This implies $\operatorname{dist}\left(\partial^{c} \phi_{\infty}\left(x_{\infty}\right), c\right.$-Cut $\left.\left(x_{\infty}\right)\right)=0$, which contradicts Theorem 5.1, and completes the proof.

Corollary 5.3 (Regularity of optimal maps). Let $M, \bar{M}$, c be as in Theorem 5.1. Assume that $\mu$ and $\nu$ are two probability measures absolutely continuous with respect to the volume measure, and whose densities are bounded away from zero and infinity. Then the unique optimal map $T$ from $\mu$ to $v$ is injective and continuous. Furthermore, if both densities are $C^{\alpha} / C^{\infty}$, then $T$ is $C^{1, \alpha} / C^{\infty}$.

Remark 5.4. The $C^{1, \alpha}$-regularity result $\left(C^{2, \alpha}\right.$ for the potential $\left.\phi\right)$ in this corollary is a direct consequence of the injectivity and continuity of $T$ applied to the theory of Liu, Trudinger and Wang [LTW]. The higher regularity $C^{\infty}$ follows from Schauder estimates.

Proof of Corollary 5.3. We recall that, under the assumption that $\mu$ and $v$ have densities bounded away from zero and infinity, there exists a $c$-convex function $\phi$ such that $T(x)=$ $c$-Exp ${ }_{x}(\nabla \phi(x))$ a.e., and $\phi$ satisfies Assumption 2.10 (see for instance [MTW] or [FKM1, Lemma 3.1]). Hence it suffices to prove that $\phi$ is $C^{1}$ and strictly $c$-convex, in the sense that $S(\bar{x})=\partial^{\bar{c}} \bar{\phi}(\bar{x})$ is a singleton for every $\bar{x} \in \partial^{c} \phi(M)=M$.

To this end, we observe that once we know that $\phi$ is strictly $c$-convex, then we can localize the proof of the $C^{1}$ regularity in [FKM1] to obtain the desired result. Thus we only need to show the strict $c$-convexity of $\phi$.

Fix $\bar{x} \in M$. By Theorem 5.1 we know that $S(\bar{x}) \subset M(\bar{x})$, so that in a neighborhood of $S(\bar{x})$ we can consider the change of coordinates $x \mapsto q=-\bar{D} c(x, \bar{x}) \in T_{\bar{x}}^{*} \bar{M}$. As shown in [FKM1, Theorem 4.3], thanks to Loeper's maximum principle (DASM) the set $S(\bar{x})$ is convex in these coordinates. Moreover, since now the cost is smooth in a neighborhood of $S(\bar{x})$, by [FKM1, Theorem 7.1 and Remark 7.2] the compact convex set $S(\bar{x})$ in the new coordinates has no exposed points on the support of $\left|\partial^{c} \phi\right|{ }^{2}$ Since in our case the support of $\left|\partial^{c} \phi\right|$ is the whole $M$, the only possibility left is that $S(\bar{x})$ is a singleton, as desired.

Sketch of the proof of Theorem 5.1. For a contradiction, assume there exists a point $\bar{x}_{0}$ such that the contact set $S\left(\bar{x}_{0}\right)$ intersects $\operatorname{Cut}\left(\bar{x}_{0}\right)$. First, we find a cut-exposed point $x_{0}$ in $S\left(\bar{x}_{0}\right) \cap \operatorname{Cut}\left(\bar{x}_{0}\right)$. More precisely we split $M$ as $M^{*} \times M^{*}$ so that $\bar{x}_{0}=\left(\bar{x}_{0}, \bar{x}_{0}^{*}\right)$,

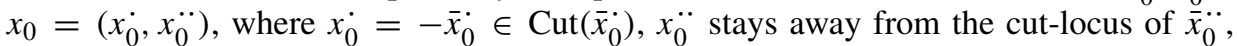
and $x_{0}^{\prime \prime}$ is an exposed point in the set $S^{\prime \prime}=\left\{y^{\prime \prime} \in M^{*} \mid\left(-\bar{x}_{0}, y^{*}\right) \in S\left(\bar{x}_{0}\right)\right\}$ (see Section 6.1). Near $\bar{x}_{0}$, for $\varepsilon \in(0,1)$ and $\delta \in[0,1]$ we construct a family of points $\bar{x}_{\varepsilon, \delta}=\left(\bar{x}_{\varepsilon}^{\cdot}, \bar{x}_{\delta}^{*}\right)$ such that $d\left(\bar{x}_{0}, \bar{x}_{\varepsilon, \delta}\right) \approx \varepsilon+\delta$, so that for $\delta$ small we have $\bar{x}_{\varepsilon, \delta} \in \bar{M}^{*}\left(x_{0}\right)$, or equivalently $x_{0} \in M^{*}\left(\bar{x}_{\varepsilon, \delta}\right)$. By suitably choosing the point $\bar{x}_{\delta}^{* *}$ in order to exploit the fact that $x_{0}^{*}$ is an exposed point for $S^{*}$, we can ensure that, if $Z_{\varepsilon, \delta, h}$ denotes a section

\footnotetext{
2 Recall that, given a compact convex set $Z \subset \mathbb{R}^{n}, z \in \partial Z$ is an exposed point if there exists a hyperplane $\Pi \subset \mathbb{R}^{n}$ such that $Z \cap \Pi=\{z\}$.
} 


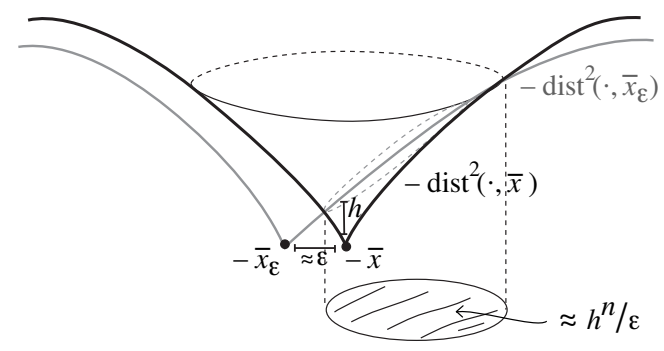

Fig. 1. On the sphere, the squared distance function from a point $\bar{x}$ looks like a cone near $-\bar{x}$. So, if $\operatorname{dist}\left(\bar{x}, \bar{x}_{\varepsilon}\right)=\operatorname{dist}\left(-\bar{x},-\bar{x}_{\varepsilon}\right) \approx \varepsilon$, the section obtained by cutting its graph with $-\operatorname{dist}^{2}\left(\cdot, \bar{x}_{\varepsilon}\right)$ at height $h$ has measure $\approx h^{n} / \varepsilon$.

obtained by cutting the graph $\phi$ with $-c\left(\cdot, \bar{x}_{\varepsilon, \delta}\right)$ at height $h$ above $x_{0}$, then for any fixed $\varepsilon \in(0,1)$ we have $Z_{\varepsilon, \delta, h} \rightarrow\left\{x_{0}\right\}$ as $\delta, h / \delta \rightarrow 0$ (see Section 6.2). In particular, for $\varepsilon>0$ fixed we have $Z_{\varepsilon, \delta, h} \subset M^{*}\left(\bar{x}_{\varepsilon, \delta}\right)$ for $\delta, h / \delta$ small (equivalently, the function $c\left(\cdot, \bar{x}_{\varepsilon, \delta}\right)$ is smooth inside $\left.Z_{\varepsilon, \delta, h}\right)$. Now we take advantage of the choice of $\bar{x}_{\varepsilon}^{\cdot}:$ on the sphere $\mathbb{S}^{n}$ the function $-\operatorname{dist}^{2}(\cdot, \bar{x})$ looks like a cone near the antipodal point $-\bar{x}$, and if $\operatorname{dist}\left(\bar{x}, \bar{x}_{\varepsilon}\right) \approx \varepsilon$ then the measure of a section obtained by cutting the graph of $-\operatorname{dist}^{2}(\cdot, \bar{x})$ with $-\operatorname{dist}^{2}\left(\cdot, \bar{x}_{\varepsilon}\right)$ at height $h$ above $-\bar{x}$ has measure $\approx h^{n} / \varepsilon$ (see Proposition 6.7). In our case, since $x_{0}^{\cdot}=-\bar{x}_{0}$, the function $\phi$ behaves like $-c\left(\cdot, \bar{x}_{0}\right) \approx-\operatorname{dist}\left(\cdot, \bar{x}_{0}\right)$ along $M \cdot$ (see Lemma 6.6). Hence by the argument above we have an improvement of a factor $1 / \varepsilon$ in the measure of $Z_{\varepsilon, \delta, h}$ (see Proposition 6.7), which allows us to show the following Alexandrov type inequality:

$$
h^{\operatorname{dim} M} \lesssim \varepsilon\left|Z_{\varepsilon, \delta, h}\right|\left|\partial^{c} \phi\left(Z_{\varepsilon, \delta, h}\right)\right| \quad \text { for } \delta \text { and } h / \delta \text { sufficiently small, }
$$

where $\lesssim$ is independent of $\varepsilon, \delta$ and $h$ (see Theorem 6.4). ${ }^{3}$ Thanks to Assumption 2.10, the above inequality implies

$$
h^{\operatorname{dim} M} \lesssim \frac{\varepsilon}{\lambda}\left|Z_{\varepsilon, \delta, h}\right|^{2} \quad \text { for } \delta \text { and } h / \delta \text { sufficiently small. }
$$

On the other hand, since $Z_{\varepsilon, \delta, h} \subset M^{*}\left(\bar{x}_{\varepsilon, \delta}\right)$ for $\delta$ and $h / \delta$ small enough, we can apply Lemma 4.1 to $Z_{\varepsilon, \delta, h}$ and have

$$
\begin{aligned}
& \lambda\left|Z_{\varepsilon, \delta, h}\right|^{2} \\
& \leq C(n)\left[\frac{\max _{x \in Z_{\varepsilon, \delta, h}}\left|\operatorname{det}\left(-D_{x} D_{\bar{x}} c\left(x, \bar{x}_{\varepsilon, \delta}\right)\right)\right|}{\min _{x \in Z_{\varepsilon, \delta, h}}\left|\operatorname{det}\left(-D_{x} D_{\bar{x}} c\left(x, \bar{x}_{\varepsilon, \delta}\right)\right)\right|}\right]^{2}\left[\sup _{x \in Z_{\varepsilon, \delta, h}} \sup _{p^{\prime} \in M^{*}(x)}\left|d c-\operatorname{Exp}_{x}\right|_{p=p^{\prime}} \mid\right] h^{\operatorname{dim} M} .
\end{aligned}
$$

3 Although this is the informal idea, the actual proof is much more involved. In particular, for technical reasons, we will also need to split $M$ as $M^{\prime} \times M^{\prime \prime}$ so that $\partial^{c} \phi\left(x_{0}\right) \cap\left(\left\{-x_{0}^{\prime}\right\} \times M^{\prime \prime}\right) \neq \emptyset\left(M^{\prime}\right.$ corresponds to the "cut-locus components") and $\partial^{c} \phi\left(x_{0}\right) \cap\left(M^{\prime} \times\left\{-x_{0}^{\prime \prime}\right\}\right)=\emptyset\left(M^{\prime \prime}\right.$ corresponds to the "regular components"); see Section 6.1. Observe that $M^{*} \subset M^{\prime}$. Then, to prove Theorem 6.4, a different argument has to be used depending on the kind of components (see Sections 6.4 and 6.5 respectively). 
The convergence $Z_{\varepsilon, \delta, h} \rightarrow\left\{x_{0}\right\}$ as $\delta, h / \delta \rightarrow 0$ further reduces this inequality to

$$
\lambda\left|Z_{\varepsilon, \delta, h}\right|^{2} \lesssim h^{\operatorname{dim} M} \quad \text { for } \delta \text { and } h / \delta \text { sufficiently small, }
$$

which contradicts (5.2) as $\varepsilon \rightarrow 0$ and completes the proof.

The rest of the paper is devoted to fleshing out the details of the above proof.

\section{Proof of Theorem 5.1}

\subsection{Cut-exposed points of contact sets}

Assume for a contradiction that there exists $\bar{x}_{0}=\left(\bar{x}_{0}^{1}, \ldots, \bar{x}_{0}^{k}\right) \in \bar{M}=M=M^{1} \times \cdots$ $\times M^{k}$ such that $S\left(\bar{x}_{0}\right) \cap c$-Cut $\left(\bar{x}_{0}\right) \neq \varnothing$. To prove Theorem 5.1 a first step is to find a $c u t$ exposed point of the contact set in the intersection with the cut-locus, which we define in the present section.

Let $y \in S\left(\bar{x}_{0}\right) \cap c$-Cut $\left(\bar{x}_{0}\right)$, and note that one of the components of $y=\left(y^{1}, \ldots, y^{k}\right)$, say $y^{j}$, satisfies $y^{j}=-\bar{x}_{0}^{j}$. Moreover we cannot have $y=-\bar{x}_{0}$. Indeed, it is not difficult to see that, if $\bar{x}_{0} \in \partial^{c} \phi\left(-\bar{x}_{0}\right)$, then $\partial^{c} \phi\left(-\bar{x}_{0}\right)=M$ (see for instance Lemma 6.6(1) below), which contradicts Assumption 2.10.

Among all points $y \in S\left(\bar{x}_{0}\right) \cap c$-Cut $\left(\bar{x}_{0}\right)$, choose one such that the number $a_{0}$ of its antipodal (or cut-locus) components is maximal, and denote the point by $y_{0}$. By rearranging the product $M^{1} \times \cdots \times M^{k}$, we may write without loss of generality that

$y_{0}=\left(-\bar{x}_{0}^{1}, \ldots,-\bar{x}_{0}^{a_{0}}, y_{0}^{a_{0}+1}, \ldots, y_{0}^{k}\right), \quad y_{0}^{j} \notin c-\operatorname{Cut}\left(\bar{x}_{0}^{j}\right) \quad \forall j=a_{0}+1, \ldots, k$.

For convenience, write

$$
M^{\cdot}=\bar{M}^{\cdot}=M^{1} \times \cdots \times M^{a_{0}}, \quad M^{\cdot}=\bar{M}^{\cdot}=M^{a_{0}+1} \times \cdots \times M^{k} .
$$

The expressions $A^{*}, A^{*}$ will be used to denote things defined for elements in $M^{*}, M^{*}$, respectively. For example,

$$
\begin{gathered}
y^{\cdot}=\left(y^{1}, \ldots, y^{a_{0}}\right), \quad y^{\cdot}=\left(y^{a_{0}+1}, \ldots, y^{k}\right), \\
c^{\cdot}\left(y^{\cdot}, \bar{y}^{\cdot}\right)=\sum_{i=1}^{a_{0}} c^{i}\left(y^{i}, \bar{y}^{i}\right), \quad c^{\cdot}\left(y^{\cdot}, \bar{y}^{\cdot}\right)=\sum_{i=a_{0}+1}^{k} c^{i}\left(y^{i}, \bar{y}^{i}\right) .
\end{gathered}
$$

Consider the set

$$
S^{\cdot}=\left\{y^{*} \in M^{\cdot} \mid\left(-\bar{x}_{0}, y^{\cdot}\right) \in S\left(\bar{x}_{0}\right)\right\}
$$

Notice that due to maximality of $a_{0}, S^{*} \subset M^{*}\left(\bar{x}_{0}^{*}\right)$ and it is embedded to $\bar{M}^{\cdot * *}\left(\bar{x}_{0}^{*}\right)$

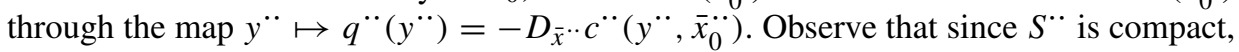
the resulting set, say $\tilde{S}^{\cdot}$, is compact too. Moreover $\tilde{S}^{\cdot}$ is convex since it is the restriction of the convex set $\tilde{S}\left(\bar{x}_{0}\right)$ to $M^{* *}\left(\bar{x}_{0}^{* *}\right)$, where $\tilde{S}\left(\bar{x}_{0}\right)$ is the image of $S\left(\bar{x}_{0}\right)$ under the map $x \mapsto-D_{\bar{x}} c\left(x, \bar{x}_{0}\right)$. (More precisely, this set $\tilde{S}\left(\bar{x}_{0}\right)$ is defined as the closure of the image 
of $S\left(\bar{x}_{0}\right) \backslash c$-Cut $\left(\bar{x}_{0}\right)$.) This compact convexity ensures the existence of an exposed point $q_{0} \cdot$ for $\tilde{S}^{\cdot}$, that is, there exists an affine function $L$ on $T_{\bar{x}_{0}^{*}}^{*} \bar{M}^{\cdot}$ such that

$$
L\left(q_{0} \ddot{0}\right)=0, \quad \text { and } L(\ddot{q})<0 \quad \forall q \ddot{*} \in \tilde{S}^{\cdot} \backslash\left\{q_{0} \ddot{ }\right\} .
$$

(In case $\tilde{S}^{\cdot}=\left\{\ddot{q_{0}}\right\}$ let $L \equiv 0$.) Note that if $L$ is such an affine function, then so is $t L$ for any $t>0$. Let $x_{0} \in S\left(\bar{x}_{0}\right)$ be the point corresponding to $q_{0}^{\ddot{*}}$ in $M$, that is,

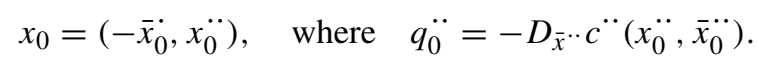

We call $x_{0}$ a cut-exposed point of $S\left(\bar{x}_{0}\right)$, since its components are either cut-locus type or exposed.

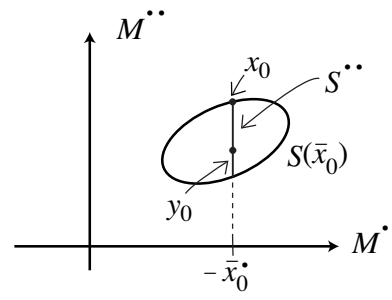

Fig. 2. Starting from a point $y_{0} \in S\left(\bar{x}_{0}\right)$ such that the number of its antipodal (or cut-locus)

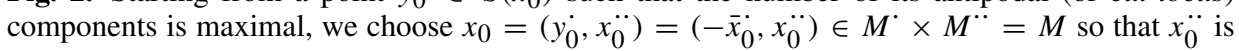
an exposed point for $S^{*}$ (in some suitable system of coordinates).

One can assume with a further rearrangement of the product $M^{\cdot}=M^{a_{0}+1} \times \cdots \times M^{k}$ that there exists $b_{0} \in\left\{a_{0}, \ldots, k\right\}$ with the following two properties:

1. For each $i_{1} \in\left\{a_{0}+1, \ldots, b_{0}\right\}$, there exists $\bar{y}_{i_{1}} \in \partial^{c} \phi\left(x_{0}\right)$ with

$$
\bar{y}_{i_{1}}^{i_{1}}=-x_{0}^{i_{1}} .
$$

2. For every $\bar{y} \in \partial^{c} \phi\left(x_{0}\right)$,

$$
\begin{aligned}
& \bar{y}^{j} \neq-x_{0}^{j} \quad\left(\text { or equivalently } \bar{y}^{j} \notin \bar{c} \text {-Cut }\left(x_{0}^{j}\right)=\operatorname{Cut}\left(x_{0}^{j}\right)\right) \quad \text { for } j=b_{0}+1, \ldots, k . \\
& \text { (If } b_{0}=a_{0},\left\{a_{0}+1, \ldots, b_{0}\right\}=\emptyset \text {.) }
\end{aligned}
$$

After this rearrangement, define

$$
M^{\prime}=\bar{M}^{\prime}=M^{1} \times \cdots \times M^{b_{0}}, \quad M^{\prime \prime}=\bar{M}^{\prime \prime}=M^{b_{0}+1} \times \cdots \times M^{k} .
$$

The expressions $A^{\prime}, A^{\prime \prime}$ will be used to denote things defined for elements in $M^{\prime}, M^{\prime \prime}$, respectively. For example,

$$
\begin{aligned}
& y=\left(y^{\prime}, y^{\prime \prime}\right), \quad \bar{y}=\left(\bar{y}^{\prime}, \bar{y}^{\prime \prime}\right) \in M=M^{\prime} \times M^{\prime \prime}=\bar{M}^{\prime} \times \bar{M}^{\prime \prime}, \\
& c(y, \bar{y})=c^{\prime}\left(y^{\prime}, \bar{y}^{\prime}\right)+c^{\prime \prime}\left(y^{\prime \prime}, \bar{y}^{\prime \prime}\right),
\end{aligned}
$$


and we have the identification

$$
T_{\bar{x}_{0}}^{*} \bar{M}=T_{\bar{x}_{0}^{\prime}}^{*} \bar{M}^{\prime} \times T_{\bar{x}_{0}^{\prime \prime}}^{*} \bar{M}^{\prime \prime}, \quad \bar{M}^{*}\left(\bar{x}_{0}\right)=\bar{M}^{\prime *}\left(\bar{x}_{0}^{\prime}\right) \times \bar{M}^{\prime \prime *}\left(\bar{x}_{0}^{\prime \prime}\right) .
$$

In the following, $n^{\prime}=\operatorname{dim} M^{\prime}, n^{\prime \prime}=\operatorname{dim} M^{\prime \prime}$ and $\pi^{\prime}, \pi^{\prime \prime}$ denote the canonical projections from $M$ to $M^{\prime}, M^{\prime \prime}$, respectively. This splitting of $M$ as $M^{\prime} \times M^{\prime \prime}$ will be important later, as in the proof of Theorem 6.4 we will need different arguments on $M^{\prime}$ and $M^{\prime \prime}$ (see Sections 6.4 and 6.5).

\subsection{Analysis near the cut-exposed point}

In this subsection we construct a family of $c$-sections $Z_{\varepsilon, \delta, h}$ of $\phi$ near the cut-exposed point $x_{0}$ defined in (6.3). Regarding these $c$-sections, two important results (Propositions 6.2 and 6.3) are obtained. In later subsections we will show an Alexandrov type inequality for $Z_{\varepsilon, \delta, h}$, which will be paired with the other Alexandrov type inequality (4.1) to yield a contradiction to the existence of such $x_{0}$, thus finishing the proof of Theorem 5.1.

Recall the affine function $L$ on $T_{\bar{x}_{0}}^{*} \bar{M}^{\cdot}$ given in (6.2). After modifying $L$ by multiplying it by an appropriate positive constant, there exists a geodesic curve $[0,1] \ni \delta \mapsto$ $\bar{x}_{\delta}^{. *} \in \bar{M} \cdot\left(x_{0}^{*}\right)$ starting from $\bar{x}_{0}^{. *}$ such that for the linear map $\nabla L$ on $T_{\bar{x}}^{*} . \bar{M}^{*}$,

$$
\nabla L\left(q^{\cdot}-\ddot{q_{0}}\right)=\left\langle\left.\frac{\partial}{\partial t}\right|_{t=0} \bar{x}_{t}^{. \cdot}, \dot{q}-q_{0}^{. \cdot}\right\rangle .
$$

Consider a $c^{\cdot}$-segment $[0,1] \ni \varepsilon \mapsto \bar{x}_{\varepsilon}^{\cdot} \in \bar{M}^{\cdot}$ with respect to $x_{0}^{\cdot}$ connecting the point $\bar{x}_{0}$. to its antipodal point $\bar{x}_{1 / 2}=x_{0}$ and then to $\bar{x}_{1}=\bar{x}_{0} \cdot\left(\bar{x}_{\varepsilon}\right.$ is nothing else than a closed geodesic starting from $\bar{x}_{0}$ and passing through $x_{0}^{\cdot}=-\bar{x}_{0}$ at $\varepsilon=1 / 2$.) Define

$$
\bar{x}_{\varepsilon, \delta}:=\left(\bar{x}_{\varepsilon}^{\cdot}, \bar{x}_{\delta}^{\cdot *}\right) \in M=M \times M^{*} .
$$

Obviously $\bar{x}_{0,0}=\bar{x}_{0}$. Two important properties follow:

(a) Since $\bar{x}_{\varepsilon}^{\cdot} \in \bar{M}^{\cdot}\left(x_{0}^{\cdot}\right)$ for $\varepsilon \in(0,1)$ and $\bar{x}_{0}^{\cdot *} \in \bar{M}^{*}\left(x_{0}^{*}\right)$ we have

$$
x_{0} \in M\left(\bar{x}_{\varepsilon, \delta}\right)=M \cdot\left(\bar{x}_{\varepsilon}^{\cdot}\right) \times M^{*}\left(\bar{x}_{\delta}^{*}\right) \quad \forall 0<\varepsilon<1, \delta \geq 0 \text { small. }
$$

(b) Since $\bar{x}_{\varepsilon, \delta}^{\cdot}=\bar{x}_{\varepsilon}^{\cdot} \neq \bar{x}_{0}^{\cdot}$ for $\varepsilon \in(0,1), \bar{x}_{1}^{\cdot}=\bar{x}_{0}$, and $\bar{x}_{0}^{\cdot}=-x_{0}^{\cdot}$, for every $\varepsilon \in(0,1)$ and $\delta \in[0,1]$ we have from Assumption 2.5, for each $c^{i}$,

$$
-c^{\cdot}\left(x^{\cdot}, \bar{x}_{\varepsilon, \delta}^{\cdot}\right)+c^{\cdot}\left(x_{0}^{\cdot}, \bar{x}_{\varepsilon, \delta}^{\cdot}\right) \leq-c^{\cdot}\left(x^{\cdot}, \bar{x}_{0}^{\cdot}\right)+c^{\cdot}\left(x_{0}^{\cdot}, \bar{x}_{0}^{\cdot}\right) \quad \forall x^{\cdot} \in M^{\cdot}
$$

with equality only when $x^{\cdot}=x_{0}$. (See for instance Lemma 6.5 below.)

Consider now the $c$-section $Z_{\varepsilon, \delta, h}$ obtained by cutting the graph of $\phi$ by the graph of $-c\left(\cdot, \bar{x}_{\varepsilon, \delta}\right)+c\left(x_{0}, \bar{x}_{\varepsilon, \delta}\right)+h$, that is,

$$
Z_{\varepsilon, \delta, h}:=\left\{x \in M \mid \phi(x)-\phi\left(x_{0}\right)+c\left(x, \bar{x}_{\varepsilon, \delta}\right)-c\left(x_{0}, \bar{x}_{\varepsilon, \delta}\right) \leq h\right\} .
$$


As can be easily seen by moving down the graph of $-c\left(\cdot, \bar{x}_{\varepsilon, \delta}\right)$ and lifting it up until it touches the graph of $\phi, \bar{x}_{\varepsilon, \delta} \in \partial \phi\left(Z_{\varepsilon, \delta, h}\right)$. Hence, thanks to Loeper's maximum principle (DASM) we have

$$
\bar{x}_{\varepsilon, \delta} \in \partial^{c} \phi\left(Z_{\varepsilon, \delta, h}\right)
$$

Proposition 6.1. The following equality holds:

$$
Z_{\varepsilon, 0,0}=S\left(\bar{x}_{\varepsilon, 0}\right)=S\left(\bar{x}_{0}\right) \cap\left(\left\{x_{0}^{\cdot}\right\} \times M^{*}\right) .
$$

Proof. From (6.8),

$$
\begin{aligned}
& \phi(x)-\phi\left(x_{0}\right)+c\left(x, \bar{x}_{\varepsilon, 0}\right)-c\left(x_{0}, \bar{x}_{\varepsilon, 0}\right) \\
& =\phi(x)-\phi\left(x_{0}\right)+c^{\cdot}\left(x^{\cdot}, \bar{x}_{\varepsilon, 0}^{\cdot}\right)-c^{\cdot}\left(x_{0}^{\cdot}, \bar{x}_{\varepsilon, 0}\right)+c^{*}\left(x^{*}, \bar{x}_{0}^{*}\right)-c^{*}\left(x_{0}^{*}, \bar{x}_{0}^{* *}\right) \\
& \geq \phi(x)-\phi\left(x_{0}\right)+c^{\cdot}\left(x^{\cdot}, \bar{x}_{0}^{\cdot}\right)-c \cdot\left(x_{0}^{\cdot}, \bar{x}_{0}\right)+c \cdot\left(x^{*}, \bar{x}_{0}^{\cdot}\right)-c^{*}\left(x_{0} \cdot \bar{x}_{0}^{*}\right) \\
& =\phi(x)-\phi\left(x_{0}\right)+c\left(x, \bar{x}_{0}\right)-c\left(x_{0}, \bar{x}_{0}\right) \geq 0 \text {. }
\end{aligned}
$$

This, together with the equality case for (6.8), yields (6.11).

The following two propositions are essential in our proof of Theorem 5.1.

Our first result shows that, for fixed $\varepsilon$, the sections $Z_{\varepsilon, \delta, h}$ converge to the point $x_{0}$ (in the sense of Kuratowski) as $\delta, h / \delta \rightarrow 0$.

Proposition 6.2. Fix $0<\varepsilon<1$. Then, for any sequences $\delta_{i}, h_{i} \rightarrow 0$ with $h_{i} / \delta_{i} \rightarrow 0$,

$$
Z_{\varepsilon, \delta_{i}, h_{i}} \rightarrow\left\{x_{0}\right\} \quad \text { as } i \rightarrow \infty .
$$

Proof. Fix arbitrary sequences $\delta_{i}, h_{i} / \delta_{i} \rightarrow 0$, and denote

$$
\begin{array}{r}
Z_{\infty}=\lim _{i \rightarrow \infty} Z_{\varepsilon, \delta_{i}, h_{i}}=\left\{z_{\infty} \in M \mid \text { there exists a sequence } z_{i} \in Z_{\varepsilon, \delta_{i}, h_{i}}\right. \\
\text { with } \left.z_{i} \rightarrow z_{\infty} \in M\right\} .
\end{array}
$$

By continuity, $z_{\infty} \in Z_{\varepsilon, 0,0}$ for each $z_{\infty} \in Z_{\infty}$, and thus by (6.11), $z_{\infty}=x_{0}$.

To show $z_{\infty}=x_{0}^{*}$, we first let $\delta>0$ be sufficiently small and fix a small (closed) neighborhood, say $U$, of $x_{0}$ so that all the derivatives (up to the second order) of the function $U \times[0,1] \ni(x, t) \mapsto c\left(x, \bar{x}_{\varepsilon, t \delta}\right)$ are uniformly bounded. Then, for $x \in Z_{\varepsilon, \delta, h} \cap U$ the following inequalities hold:

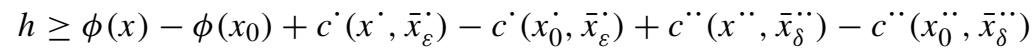

$$
\begin{aligned}
& \geq-c^{\cdot}\left(x^{\cdot}, \bar{x}_{0}^{\cdot}\right)+c^{\cdot}\left(x_{0}^{\cdot}, \bar{x}_{0}^{\cdot}\right)-c^{*}\left(x^{\cdot *}, \bar{x}_{0}^{*}\right)+c^{*}\left(x_{0}^{\cdot}, \bar{x}_{0}^{*}\right) \\
& \left.+c^{\cdot}\left(x^{\cdot}, \bar{x}_{\varepsilon}^{\cdot}\right)-c^{\cdot}\left(x_{0}^{\cdot}, \bar{x}_{\varepsilon}^{\cdot}\right)+c^{*}\left(x^{*}, \bar{x}_{\delta}^{*}\right)-c^{*}\left(x_{0}^{\cdot}, \bar{x}_{\delta}^{*}\right) \quad \text { (since } \bar{x}_{0} \in \partial^{c} \phi\left(x_{0}\right)\right)
\end{aligned}
$$

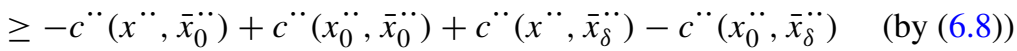

$$
\begin{aligned}
& \geq\left\langle D_{\bar{x}} c^{*}\left(x^{*}, \bar{x}_{0}^{*}\right)-D_{\bar{x}} c^{\cdot \cdot}\left(x_{0}^{*}, \bar{x}_{0}^{*}\right),\left.\frac{\partial}{\partial t}\right|_{t=0} \bar{x}_{t \delta}\right\rangle+O\left(\delta^{2}\right) \\
& =\delta \nabla L\left(D_{\bar{x}} c^{\cdot}\left(x^{*}, \bar{x}_{0} \cdot\right)-D_{\bar{x}} c^{\cdot}\left(x_{0}, \bar{x}_{0}\right)\right)+O\left(\delta^{2}\right) \quad(\text { by }(6.6)) \text {. }
\end{aligned}
$$


Use the coordinate $q^{*}\left(x^{*}\right)=-D_{\bar{x}} \cdot c^{*}\left(x^{*}, \bar{x}_{0}^{* *}\right)$ to rewrite this as

$$
\nabla L\left(q \ddot{q}-\ddot{q_{0}}\right) \geq-h / \delta-O(\delta) .
$$

Since $L\left(q_{0} \ddot{)}\right)=0$ this gives

$$
L\left(q \ddot{*}-\ddot{q_{0}}\right) \geq-h / \delta-O(\delta) .
$$

Consider now the sequences $\delta_{i}, h_{i} / \delta_{i} \rightarrow 0$, and any convergent subsequence of $z_{i} \in$ $Z_{\varepsilon, \delta_{i}, h_{i}} \cap U$. For the limit $z_{\infty}$, let $q^{\cdot \cdot}=-D_{\bar{x} \cdot .} c\left(z_{\infty}^{\cdot}, \bar{x}_{0}^{*}\right)$. Then $q_{\infty} \in \tilde{S}^{\cdot}$ (since $z_{\infty} \in$ $Z_{\varepsilon, 0,0} \subset S\left(\bar{x}_{0}\right)$ by (6.11)), and from the above inequality we get

$$
L\left(\ddot{q_{\infty}}-\ddot{q_{0}}\right) \geq 0,
$$

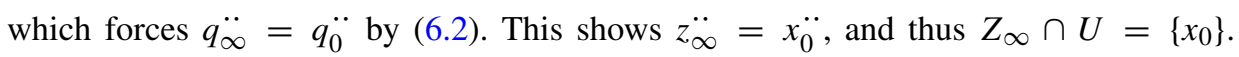
To finish the proof notice that each $Z_{\varepsilon, \delta, h}$ is connected, and so is the limit $Z_{\infty}$. (The connectedness can be seen by noticing that the set $Z_{\varepsilon, \delta, h}$ is convex in the coordinates $q(x)=-D_{\bar{x}} c\left(x, \bar{x}_{\varepsilon, \delta}\right) \in T_{\bar{x}_{\varepsilon, \delta}^{*}}^{*} \bar{M}$.) Therefore $Z_{\infty}=\left\{x_{0}\right\}$, as desired.

Proposition 6.3. There exists $\delta_{0}=\delta_{0}(\varepsilon)>0$ such that, if $0 \leq \delta \leq \delta_{0}, 0 \leq h \leq \delta^{2}$, then for each $\bar{y}=\left(\bar{y}^{\prime}, \bar{y}^{\prime \prime}\right) \in \partial^{c} \phi\left(Z_{\varepsilon, \delta, h}\right)$ the component $\bar{y}^{\prime \prime}$ stays away from the cutlocus of the component $z^{\prime \prime}$ of $z$ (i.e., $\left.\bar{y}^{\prime \prime} \in \bar{M}^{\prime \prime}\left(z^{\prime \prime}\right)\right)$ for every $z \in Z_{\varepsilon, \delta, h}$. Equivalently $\bar{\pi}^{\prime \prime}\left(\partial^{c} \phi\left(Z_{\varepsilon, \delta, h}\right)\right) \subset \bigcap_{z \in Z_{\varepsilon, \delta, h}} \bar{M}^{\prime \prime}\left(z^{\prime \prime}\right)$.

Proof. Suppose the statement is false along some sequence $\delta_{i}, h_{i} \rightarrow 0$ with $h_{i} \leq \delta_{i}^{2}$, and let $x_{i}, z_{i} \in Z_{\varepsilon, \delta, h}, \bar{y}_{i} \in \partial^{c} \phi\left(x_{i}\right)$ be such that $\bar{y}_{i}^{\prime \prime} \in \operatorname{Cut}\left(z_{i}^{\prime \prime}\right)$. Since $Z_{\varepsilon, \delta_{i}, h_{i}} \rightarrow\left\{x_{0}\right\}$, both $x_{i}, z_{i}$ tend to $x_{0}$. Moreover if $\bar{y}_{\infty}$ is a cluster point for $\left\{\bar{y}_{i}\right\}_{i \in \mathbb{N}}$, then $\bar{y}_{\infty} \in \partial^{c} \phi\left(x_{0}\right)$ and $\bar{y}_{\infty}^{\prime \prime} \in \operatorname{Cut}\left(x_{0}^{\prime \prime}\right)$. This contradicts the choice of $M^{\prime \prime}$ (see (6.5)) and concludes the proof.

\subsection{An Alexandrov type estimate near the cut-exposed point}

We state the main theorem to be proved in the rest of the paper.

Theorem 6.4 (Alexandrov upper bound near cut-exposed point). Fix $0<\varepsilon<1$, and let $Z_{\varepsilon, \delta, h}$ be as in (6.9). There exists $\delta_{1}=\delta_{1}(\varepsilon)>0$ so that, if $0<\delta \leq \delta_{1}$, then there exists $h_{1}=h_{1}(\varepsilon, \delta)$ such that

$$
h^{\operatorname{dim} M} \lesssim \varepsilon^{a_{0}}\left|Z_{\varepsilon, \delta, h}\right|\left|\partial^{c} \phi\left(Z_{\varepsilon, \delta, h}\right)\right| \quad \forall 0<h \leq h_{1}(\varepsilon, \delta),
$$

where $\lesssim$ is independent of $\varepsilon, \delta$ and $h$.

This result concludes the proof of Theorem 5.1, since for $\varepsilon>0$ small enough and $\delta, h / \delta \rightarrow 0$ we have $Z_{\varepsilon, \delta, h} \rightarrow\left\{x_{0}\right\}$ (by Proposition 6.2), and (6.12) is in contradiction with (4.1).

The following subsections are devoted to the proof of Theorem 6.4, which we divide into three parts. First, in Section 6.4 we get Alexandrov type estimates for the sets obtained by the intersection of $Z_{\varepsilon, \delta, h}$ with the cut-locus components of $x_{0}$. In Section 6.5, we analyze the projection $\pi^{\prime \prime}\left(Z_{\varepsilon, \delta, h}\right)$ of $Z_{\varepsilon, \delta, h}$ onto the regular component $M^{\prime \prime}$ of $x_{0}$. We construct a suitable convex set, say $\tilde{C}$, which has size comparable to the image $\partial^{c} \phi\left(Z_{\varepsilon, \delta, h}\right)$, and we get a version of the estimate (6.12) involving $\tilde{C}$ and $\pi^{\prime \prime}\left(Z_{\varepsilon, \delta, h}\right)$ (see Proposition 6.8(3)). Finally in Section 6.6 we combine these results and conclude the proof. 


\subsection{Proof of Theorem 6.4: analysis in the cut-locus component $M^{\prime}$}

The main result of this section is Proposition 6.7 that gives an Alexandrov type estimate for the intersection of $Z_{\varepsilon, \delta, h}$ with the cut-locus components of $x_{0}$.

We start with a few elementary results.

Lemma 6.5. Let $\mathbb{S}^{n}$ be the standard round sphere, and $c(x, \bar{x})=f(\operatorname{dist}(x, \bar{x}))$ for $(x, \bar{x}) \in \mathbb{S}^{n} \times \mathbb{S}^{n}$, where $f$ is a smooth strictly increasing function $f: \mathbb{R}_{+} \rightarrow \mathbb{R}_{+}$. Assume that c satisfies Assumption 2.5. Then, for every $x, \bar{x} \in \mathbb{S}^{n}$,

$$
-c(-\bar{x}, \bar{y})+c(-\bar{x}, \bar{x}) \geq-c(x, \bar{y})+c(x, \bar{x}) \quad \forall \bar{y} \in \mathbb{S}^{n},
$$

where $-\bar{x}$ denotes the antipodal point of $\bar{x}$. Moreover equality holds if and only if $\bar{y}=\bar{x}$.

Proof. For any $x, \bar{x} \in \mathbb{S}^{n}$, one can find a $c$-segment $x(s)$ with respect to $\bar{x}$ such that $x(0)=x(1)=-\bar{x}$ and $x\left(s_{0}\right)=x$ for some $s_{0} \in[0,1]$. The inequality (together with the characterization of the equality case) then follows from $\left(\mathbf{D A S M} \mathbf{S}^{+}\right)$for the function $\bar{m}_{s}(\cdot)=-c(x(s), \cdot)+c(x(s), \bar{x})$.

For each $1 \leq i \leq k$ and $z \in M$, let $M_{z}^{i}$ denote the $i$ th slice of $M$ through $z$, that is,

$$
M_{z}^{i}:=\left\{x \in M \mid x^{j}=z^{j} \text { for } j \neq i\right\} .
$$

The following lemma generalizes the fact that on $M=\bar{M}=\mathbb{S}^{n}$ with $c=\operatorname{dist}^{2} / 2$, if $x \in \mathbb{S}^{n}$ and $-x \in \partial^{c} \phi(x)$, then $\partial^{c} \phi(x)=\mathbb{S}^{n}$.

Lemma 6.6. Let $M, \bar{M}, c$ be as in Theorem 5.1. Let $\phi$ be a c-convex function on M. Fix $z=\left(z^{1}, \ldots, z^{k}\right) \in M=M^{1} \times \cdots \times M^{k}$ and an open set $U$ with $z \in U$. Fix $i \in\{1, \ldots, k\}$, and let $\bar{z} \in M$ with $\bar{z}^{i}=-z^{i}$. The following holds:

(1) If $\bar{z} \in\left[\partial^{c} \phi(U)\right]_{z}$ (resp. $\left.\bar{z} \in \partial^{c} \phi(z)\right)$, then $M_{\bar{z}}^{i} \subset\left[\partial^{c} \phi(U)\right]_{z}\left(\operatorname{resp} . M_{\bar{z}}^{i} \subset \partial^{c} \phi(z)\right)$.

(2) Suppose $\bar{z} \in \partial^{c} \phi(z)$. Then, for each $x \in M_{z}^{i}, \phi(x)-\phi(z)=-c^{i}\left(x^{i},-z^{i}\right)+$ $c^{i}\left(z^{i},-z^{i}\right)$.

Proof. To prove (1) it is enough to observe that for $\bar{x} \in M_{\bar{z}}^{i}$ and $x \in M$,

$$
\begin{aligned}
& -c(x, \bar{x})+c(z, \bar{x})=-c^{i}\left(x^{i}, \bar{x}^{i}\right)+c\left(z^{i}, \bar{x}^{i}\right)+\sum_{j \neq i}\left[-c^{j}\left(x^{j}, \bar{z}^{j}\right)+c^{j}\left(x^{j}, \bar{z}^{j}\right)\right] \\
& \leq-c^{i}\left(x^{i},-z^{i}\right)+c\left(z^{i},-z^{i}\right)+\sum_{j \neq i}\left[-c^{j}\left(x^{j}, \bar{z}^{j}\right)+c^{j}\left(z^{j}, \bar{z}^{j}\right)\right] \quad \text { (by Lemma 6.5) } \\
& =-c(x, \bar{z})+c(z, \bar{z}) \quad\left(\text { since } \bar{z}^{i}=-z^{i}\right) .
\end{aligned}
$$

The last line is bounded from above by $\phi(x)-\phi(z)$ either if $x \in \partial U$ or if $\bar{z} \in \partial^{c} \phi(z)$.

Let us prove (2). Suppose $\bar{z} \in \partial^{c} \phi(z)$. By duality (Lemma 2.6), $z \in \partial^{\bar{c}} \bar{\phi}(\bar{z})$ for the dual $\bar{c}$-convex function $\bar{\phi}$. Applying (1) to $\bar{\phi}$ we get $M_{z}^{i} \in \partial^{\bar{c}} \bar{\phi}(\bar{z})$, or equivalently $M_{z}^{i} \subset S(\bar{z})$. Therefore for all $x \in M_{z}^{i}$ we have

$$
\begin{aligned}
\phi(x)-\phi(z) & =-c(x, \bar{z})+c(z, \bar{z}) \\
& =-c^{i}\left(x^{i},-z^{i}\right)+c^{i}\left(z^{i},-z^{i}\right) \quad\left(\text { since } x^{j}=z^{j} \text { for } j \neq i\right) .
\end{aligned}
$$


Let $i \in\left\{1, \ldots, b_{0}\right\}$, i.e., $M^{i}$ is a component of $M^{\prime}$. Recall that $x_{0}$ is the cut-exposed point defined in (6.3). By definition of $b_{0}$ in (6.4) and (6.5), there exists $\bar{y}_{i} \in \partial^{c} \phi\left(x_{0}\right)$ such that $\bar{y}_{i}^{i}=-x_{0}^{i}$. (If $i \leq a_{0}$ then one can choose $\bar{y}_{i}=\bar{x}_{0}$.) Let $Z_{\varepsilon, \delta, h}^{i}:=\pi^{i}\left(Z_{\varepsilon, \delta, h} \cap M_{x_{0}}^{i}\right)$ for the canonical projection $\pi^{i}: M \rightarrow M^{i}$. Then Lemma 6.6(2) implies

$$
Z_{\varepsilon, \delta, h}^{i}=\left\{x^{i} \in M^{i} \mid-c^{i}\left(x^{i},-x_{0}^{i}\right)+c^{i}\left(x_{0}^{i},-x_{0}^{i}\right)+c^{i}\left(x^{i}, \bar{x}_{\varepsilon, \delta}^{i}\right)-c^{i}\left(x_{0}^{i}, \bar{x}_{\varepsilon, \delta}^{i}\right) \leq h\right\} .
$$

Here comes the main result of this section.

Proposition 6.7. There exist $\delta_{2}=\delta_{2}(\varepsilon)>0$ such that, if $0<\delta \leq \delta_{2}$, then there exists $h_{2}=h_{2}(\varepsilon, \delta)$ such that the set $Z_{\varepsilon, \delta, h}^{i}$ satisfies the following estimates for $0<h \leq h_{2}$ :

$$
\begin{array}{ll}
h^{\operatorname{dim} M^{i}} \lesssim \varepsilon\left|Z_{\varepsilon, \delta, h}^{i}\right| & \text { if } 1 \leq i \leq a_{0}, \\
h^{\operatorname{dim} M^{i}} \lesssim\left|Z_{\varepsilon, \delta, h}^{i}\right| & \text { if } a_{0}+1 \leq i \leq b_{0},
\end{array}
$$

where $\lesssim i$ independent of $\varepsilon, \delta$ and $h$, and $\left|Z_{\varepsilon, \delta, h}^{i}\right|$ denotes the Riemannian volume in the submanifold $M^{i}$.

Proof. From (6.13) and Lemma 6.5 we have $Z_{\varepsilon, \delta, h}^{i} \rightarrow\left\{x_{0}^{i}\right\}$ as $h \rightarrow 0$. Thus for sufficiently small $h$ we can embed $Z_{\varepsilon, \delta, h}^{i}$ into $\in T_{x_{0}^{i}}^{*} M^{i}$ by $x^{i} \mapsto q^{i}\left(x^{i}\right)=-D_{\bar{x}^{i}} c^{i}\left(x^{i}, x_{0}^{i}\right)$. Let $W_{h}^{i}$ be its image. Then

$$
\left|W_{h}^{i}\right| \leq\left(\max _{x^{i} \in Z_{\varepsilon, \delta, h}^{i}}\left|-D_{x^{i}} D_{\bar{x}^{i}} c^{i}\left(x^{i}, x_{0}^{i}\right)\right|\right)\left|Z_{\varepsilon, \delta, h}^{i}\right| \lesssim\left|Z_{\varepsilon, \delta, h}^{i}\right|
$$

for $h$ sufficiently small. In the following we bound $\left|W_{h}^{i}\right|$ from below.

Without loss of generality, assume $M^{i}$ is the unit sphere. Let $q_{0}^{i}=q^{i}\left(x_{0}^{i}\right)$. By abuse of notation use $c^{i}\left(q^{i}, \bar{x}^{i}\right)$ to denote $c^{i}\left(x^{i}\left(q^{i}\right), \bar{x}^{i}\right)$, and renormalize this cost function as

$$
c_{h}^{i}\left(q^{i}, \bar{x}^{i}\right)=\frac{1}{h}\left[c^{i}\left(h q^{i}+q_{0}^{i}, \bar{x}^{i}\right)-c^{i}\left(q_{0}^{i}, \bar{x}^{i}\right)\right] .
$$

Then (6.13) implies $W_{h}^{i}=h \hat{W}_{h}^{i}+q_{0}^{i}$, where

$$
\hat{W}_{h}^{i}:=\left\{q^{i} \in T_{x_{0}^{i}}^{*} M^{i} \mid-c_{h}^{i}\left(q^{i},-x_{0}^{i}\right)+c_{h}^{i}\left(q^{i}, \bar{x}_{\varepsilon, \delta}^{i}\right) \leq 1\right\} .
$$

Recall $c^{i}=f^{i}\left(\right.$ dist $\left._{i}\right)$ for some smooth non-negative uniformly convex function $f^{i}$ : $\mathbb{R}_{+} \rightarrow \mathbb{R}_{+}$such that $f^{i}(0)=0$ and $\frac{d f^{i}}{d t}(0)=0$. Thus, as $h \rightarrow 0$ the renormalized cost $-c_{h}^{i}\left(q^{i},-x_{0}^{i}\right)$ converges to the conical function

$$
q^{i} \mapsto \frac{d f^{i}}{d t}(\pi)\left|q^{i}\right| \quad \text { for } q^{i} \in T_{x_{0}^{i}}^{*} M^{i} .
$$

(Here, we used $\operatorname{dist}_{i}\left(x_{0}^{i},-x_{0}^{i}\right)=\pi$.)

Case I: If $1 \leq i \leq a_{0}$, then $\bar{x}_{\varepsilon, \delta}^{i}=\bar{x}_{\varepsilon}^{\cdot i}$, and so $c_{h}^{i}\left(q, \bar{x}_{\varepsilon, \delta}^{i}\right)$ converges to the linear function

$$
q^{i} \mapsto D_{q} c^{i}\left(q_{0}^{i}, \bar{x}_{\varepsilon}^{\cdot i}\right) \cdot q^{i}
$$



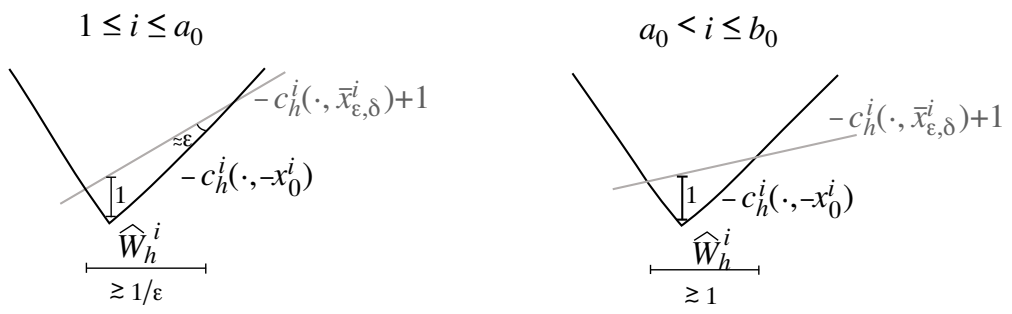

Fig. 3. If $1 \leq i \leq a_{0}$ then $-x_{0}^{i}=\bar{x}_{0}^{i}$ and $\operatorname{dist}_{i}\left(-x_{0}^{i}, \bar{x}_{\varepsilon, \delta}^{i}\right) \approx \varepsilon$, so the size of the section is of order $1 / \varepsilon$ (see also Figure 1). On the other hand, if $a_{0}<i \leq b_{0}$ then $-x_{0}^{i} \neq \bar{x}_{0}^{i}$, which implies that $\operatorname{dist}_{i}\left(-x_{0}^{i}, \bar{x}_{\varepsilon, \delta}^{i}\right)$ is uniformly bounded away from 0 , and the size of the section is of order 1 .

where

$$
\left|D_{q} c^{i}\left(q_{0}^{i}, \bar{x}_{\varepsilon}^{\cdot i}\right)\right|=\frac{d f^{i}}{d t}(\pi-2 \pi \varepsilon) \geq \frac{d f^{i}}{d t}(\pi)-C \varepsilon
$$

for some constant $C>0$. (Here, we used $\operatorname{dist}\left(x_{0}^{i}, \bar{x}_{\varepsilon}^{\cdot i}\right)=\pi-2 \pi \varepsilon$.) Therefore one can easily check that

$$
\lim _{h \rightarrow 0}\left|\hat{W}_{h}^{i}\right| \gtrsim 1 / \varepsilon
$$

and thus for $h>0$ sufficiently small,

$$
\left|W_{h}^{i}\right|=h^{\operatorname{dim} M^{i}}\left|\hat{W}_{h}^{i}\right| \gtrsim h^{\operatorname{dim} M^{i}} / \varepsilon .
$$

Case II: If $a_{0}<i \leq b_{0}$, then $\bar{x}_{\varepsilon, \delta}^{i}=\bar{x}_{\delta} \cdot{ }^{i}$. Similarly to the above case, $c_{h}^{i}\left(q, \bar{x}_{\varepsilon, \delta}^{i}\right)$ converges to the linear function

$$
q^{i} \mapsto D_{q} c^{i}\left(q_{0}^{i}, \bar{x}_{\delta}^{\cdot i}\right) \cdot q^{i} .
$$

Since $\bar{x}_{\delta}^{*} \in \bar{M}^{*}\left(x_{0}^{*}\right)$ for $\delta>0$ small enough, there exist positive constants $C, C_{0}$ such that

$$
\left|D_{q} c^{i}\left(q_{0}^{i}, \bar{x}_{\delta}^{\cdot * i}\right)\right| \leq \frac{d f^{i}}{d t}(\pi-C) \leq \frac{d f^{i}}{d t}(\pi)-C_{0},
$$

where for the last inequality we used the uniform convexity of $f$. From this one can check that $\lim _{h \rightarrow 0}\left|\hat{W}_{h}^{i}\right| \gtrsim 1$, and thus for sufficiently small $h>0$,

$$
\left|W_{h}^{i}\right| \gtrsim h^{\operatorname{dim} M^{i}}
$$

\subsection{Proof of Theorem 6.4: analysis in the regular component $M^{\prime \prime}$}

The main result of this subsection is Proposition 6.8. Fix $0<\varepsilon<1$, and assume that $\delta$ and $h / \delta$ are sufficiently small so that, as in Proposition 6.3 , the set $Z_{\varepsilon, \delta, h}$ is close to the cut-exposed point $x_{0}$, and so in particular $Z_{\varepsilon, \delta, h} \subset M\left(\bar{x}_{\varepsilon, \delta}\right)$. Consider the change of coordinates $q \in T_{\bar{x}_{\varepsilon, \delta}}^{*} \bar{M} \mapsto x(q) \in M\left(\bar{x}_{\varepsilon, \delta}\right)$ induced by the relation

$$
q=-D_{\bar{x}} c\left(x(q), \bar{x}_{\varepsilon, \delta}\right),
$$


and let $\tilde{Z}_{\varepsilon, \delta, h} \in T_{\bar{x}_{\varepsilon, \delta}^{*}}^{*} \bar{M}$ be the set $Z_{\varepsilon, \delta, h}$ in this chart. The function $\phi$ and the cost $c$ are transformed to

$$
\begin{aligned}
\varphi(q) & =\phi(x(q))+c\left(x(q), \bar{x}_{\varepsilon, \delta}\right), \\
\tilde{c}(q, \bar{y}) & =c(x(q), \bar{y})-c\left(x(q), \bar{x}_{\varepsilon, \delta}\right) \quad \text { for }(q, \bar{y}) \in T_{\bar{x}_{\varepsilon, \delta}}^{*} \bar{M} \times \bar{M} .
\end{aligned}
$$

Notice that $\tilde{c}\left(q, \bar{x}_{\varepsilon, \delta}\right) \equiv 0$, and $\varphi$ is a $\tilde{c}$-convex function on $T_{\bar{x}_{\varepsilon, \delta}^{*}}^{*} \bar{M}$. Moreover

$$
\tilde{Z}_{\varepsilon, \delta, h}=\left\{q \in T_{\bar{x}_{\varepsilon, \delta}^{*}}^{*} \mid \varphi(q)-\varphi\left(q_{0}\right) \leq h\right\}
$$

where $q_{0}$ is the point corresponding to $x_{0}$ in this new chart. It is important to recall that, thanks to Assumption 2.4 (convex DASM), $\tilde{c}$ and $\varphi$ are convex. (See Section 3.2.)

We have the natural decomposition (with obvious notation)

$$
\begin{aligned}
q=\left(q^{\prime}, q^{\prime \prime}\right) & =\left(-D_{\bar{x}^{\prime}} c^{\prime}\left(x^{\prime}\left(q^{\prime}\right), \bar{x}_{\varepsilon, \delta}^{\prime}\right),-D_{\bar{x}^{\prime \prime}} c^{\prime \prime}\left(x^{\prime \prime}\left(q^{\prime \prime}\right), \bar{x}_{\varepsilon, \delta}^{\prime \prime}\right)\right) \\
& \in T_{\bar{x}_{\varepsilon, \delta}}^{*} \bar{M}=T_{\bar{x}_{\varepsilon, \delta}^{\prime}}^{*} \bar{M}^{\prime} \times T_{\bar{x}_{\varepsilon, \delta}^{\prime \prime}}^{*} \bar{M}^{\prime \prime} .
\end{aligned}
$$

(Here, one should keep in mind that, by the definition of $\bar{x}_{\varepsilon, \delta}$, the component $\bar{x}_{\varepsilon, \delta}^{\prime \prime}$ in $M^{\prime \prime}$ does not depend on $\varepsilon$.) The modified cost $\tilde{c}(q, \bar{y})$ has the decomposition

$$
\tilde{c}(q, \bar{y})=\tilde{c}^{\prime}\left(q^{\prime}, \bar{y}^{\prime}\right)+\tilde{c}^{\prime \prime}\left(q^{\prime \prime}, \bar{y}^{\prime \prime}\right)
$$

where

$$
\begin{array}{cl}
\tilde{c}^{\prime}\left(q^{\prime}, \bar{y}^{\prime}\right)=c^{\prime}\left(x^{\prime}\left(q^{\prime}\right), \bar{y}^{\prime}\right)-c^{\prime}\left(x^{\prime}\left(q^{\prime}\right), \bar{x}_{\varepsilon, \delta}^{\prime}\right) & \text { for } q^{\prime} \in T_{\bar{x}_{\varepsilon, \delta}^{*}}^{*} \bar{M}^{\prime}, \\
\tilde{c}^{\prime \prime}\left(q^{\prime \prime}, \bar{y}^{\prime \prime}\right)=c^{\prime}\left(x^{\prime \prime}\left(q^{\prime \prime}\right), \bar{y}^{\prime \prime}\right)-c^{\prime \prime}\left(x^{\prime \prime}\left(q^{\prime \prime}\right), \bar{x}_{\varepsilon, \delta}^{\prime \prime}\right) & \text { for } q^{\prime \prime} \in T_{\bar{x}_{\varepsilon, \delta}^{\prime \prime}}^{*} \bar{M}^{\prime \prime} .
\end{array}
$$

Let $\tilde{\pi}^{\prime}, \tilde{\pi}^{\prime \prime}$ denote the canonical projection from $T_{\bar{x}_{\varepsilon, \delta}}^{*} \bar{M}$ onto $T_{\bar{x}_{\varepsilon, \delta}^{\prime}}^{*} \bar{M}^{\prime}$ and $T_{\bar{x}_{\varepsilon, \delta}^{\prime \prime}}^{*} \bar{M}^{\prime \prime}$, respectively.

Now, let us construct a convex set $\tilde{C} \subset T_{q_{0}}^{*}\left(T_{\bar{x}_{\varepsilon, \delta}}^{*} \bar{M}\right)$ that we will use later to estimate $\left|\partial^{c} \phi\left(Z_{\varepsilon, \delta, h}\right)\right|$ from below (see Proposition 6.9). The strategy of the proof follows the lines of the one of [FKM1, Proposition 6.10].

Proposition 6.8. Fix $0<\varepsilon<1$, and assume that $0<\delta \leq \delta_{0}$ and $0<h \leq \delta^{2}$, with $\delta_{0}$ as in Proposition 6.3. Then there exists a convex set $\tilde{C} \in T_{q_{0}}^{*}\left(T_{\bar{x}_{\varepsilon, \delta}}^{*} \bar{M}\right)$ with the following properties:

(1) $\tilde{C} \subset\{0\} \times T_{q_{0}^{\prime \prime}}^{*}\left(T_{\bar{x}_{\varepsilon, \delta}^{\prime \prime}}^{*} \bar{M}^{\prime \prime}\right) \subset T_{q_{0}^{\prime}}^{*}\left(T_{\bar{x}_{\varepsilon, \delta}^{\prime}}^{*} \bar{M}^{\prime}\right) \times T_{q_{0}^{\prime \prime}}^{*}\left(T_{\bar{x}_{\varepsilon, \delta}^{\prime \prime}}^{*} \bar{M}^{\prime \prime}\right)$;

(2) $\tilde{c}-\operatorname{Exp}_{q_{0}} \tilde{C}=\left\{\bar{z} \in M \mid-\partial_{q} \tilde{c}\left(q_{0}, \bar{z}\right) \cap \tilde{C} \neq \emptyset\right\} \subset\left[\partial^{c} \phi\left(Z_{\varepsilon, \delta, h}\right)\right]_{x_{0}} \subset \partial^{c} \phi\left(Z_{\varepsilon, \delta, h}\right)$, where $\partial_{q}$ denotes the subdifferential with respect to the $q$ variable;

(3) $\mathscr{H}^{n^{\prime \prime}}(\tilde{C}) \mathscr{H}^{n^{\prime \prime}}\left(\tilde{\pi}^{\prime \prime}\left(\tilde{Z}_{\varepsilon, \delta, h}\right)\right) \gtrsim h^{n^{\prime \prime}}$, where is independent of $h, \delta, \varepsilon$.

Proof. In the following, we first construct such a set $\tilde{C}$ and then we show the desired properties. The set $\tilde{C}$ will be given as the convex hull of certain covectors $\hat{p}_{1}, \ldots, \hat{p}_{n^{\prime \prime}}$ (see (6.22)). We go through several steps. 
First we find some auxiliary covectors $p_{\tilde{\tau}}, \ldots, p_{n^{\prime \prime}}$. From Lemma 3.1 applied to the convex set $\tilde{\pi}^{\prime \prime}\left(\tilde{Z}_{\varepsilon, \delta, h}\right)$, there is an ellipsoid $\tilde{E}$ such that

$$
\tilde{E} \subset \tilde{\pi}^{\prime \prime}\left(\tilde{Z}_{\varepsilon, \delta, h}\right) \subset n^{\prime \prime} \tilde{E}
$$

where the scaling $n^{\prime \prime} \tilde{E}$ is with respect to the barycenter of the ellipsoid. Let $p_{i}^{\prime \prime}, 1 \leq$ $i \leq n^{\prime \prime}$, denote the unit orthogonal covectors parallel to the axes of the ellipsoid $\tilde{E}$, and denote by $a_{i}$ the length of the $i$-th principal axis of $\tilde{E}$. Find hyperplanes $\Pi_{i}^{\prime \prime} \subset T_{\bar{x}_{\varepsilon, \delta}^{\prime \prime}}^{*} \bar{M}^{\prime \prime}$ that are orthogonal to $p_{i}^{\prime \prime}$ and touch tangentially the boundary of $\tilde{\pi}^{\prime \prime}\left(\tilde{Z}_{\varepsilon, \delta, h}\right)$ at points $q_{i}^{\prime \prime}$, $1 \leq i \leq n^{\prime \prime}$. Let $q_{0}^{\prime \prime}$ be the point in $T_{\bar{x}_{\varepsilon, \delta}^{\prime \prime}}^{*} \bar{M}^{\prime \prime}$ corresponding to $x_{0}$, and denote by $\ell_{i}$ the distance from $q_{0}^{\prime \prime}$ to $\Pi_{i}^{\prime \prime}$. Then, thanks to $(6.16)$ we have

$$
\prod_{i}^{n^{\prime \prime}} \ell_{i} \leq \prod_{i}^{n^{\prime \prime}}\left(2 n^{\prime \prime} a_{i}\right) \lesssim \mathscr{H}^{n^{\prime \prime}}\left(\tilde{\pi}^{\prime \prime}\left(\tilde{Z}_{\varepsilon, \delta, h}\right)\right)
$$

For each $q_{i}^{\prime \prime}$, there exists $q_{i}^{\prime} \in T_{\bar{x}_{\varepsilon, \delta}^{\prime}}^{*} \bar{M}^{\prime}$ such that the hyperplane $\Pi_{i}:=T_{\bar{x}_{\varepsilon, \delta}^{\prime}}^{*} \bar{M}^{\prime} \times \Pi_{i}^{\prime \prime} \subset$ $T_{\bar{x}_{\varepsilon, \delta}^{*}}^{*} \bar{M}$ tangentially touches the boundary $\partial \tilde{Z}_{\varepsilon, \delta, h}$ at the point $q_{i}=\left(q_{i}^{\prime}, q_{i}^{\prime \prime}\right)$. Let $x_{i}=$ $\bar{c}$-Exp $\bar{x}_{\varepsilon, \delta} q_{i}$. Since $p_{i}=\left(0, p_{i}^{\prime \prime}\right)$ is orthogonal to $\Pi_{i}$ and $\tilde{Z}_{\varepsilon, \delta, h}$ is a sublevel set of the convex function $\varphi$, there exists a scalar multiple $t_{i} \in \mathbb{R}_{+}$such that $t_{i} p_{i} \in \partial \varphi\left(q_{i}\right)$. By Assumption 2.2 and Loeper's maximum principle (DASM) (Lemma 2.7), the point $\bar{z}_{i}=$ $\tilde{c}$-Exp $q_{i} t_{i} p_{i}$ satisfies $\bar{z}_{i} \in \partial^{\tilde{c}} \varphi\left(q_{i}\right)=\partial^{c} \phi\left(x_{i}\right)$. Note that in fact,

$$
\bar{z}_{i}=\tilde{c}-\operatorname{Exp}_{q_{i}} t_{i} p_{i}=c-\operatorname{Exp}_{x_{i}} \eta\left(t_{i} p_{i}\right)
$$

where $\eta$ is the affine map given by Lemma 3.4 (in whose statement we replace $x_{0}, q_{0}$ and $\bar{y}_{0}$ with $x_{i}, q_{i}$ and $\bar{x}_{\varepsilon, \delta}$, respectively). Moreover, using the decomposition

$$
p_{i}=\left(0, p_{i}^{\prime \prime}\right) \in T_{q_{i}^{\prime}}^{*}\left(T_{\bar{x}_{\varepsilon, \delta}^{\prime}}^{*} \bar{M}^{\prime}\right) \times T_{q_{i}^{\prime \prime}}^{*}\left(T_{\bar{x}_{\varepsilon, \delta}^{\prime \prime}}^{*} \bar{M}^{\prime \prime}\right),
$$

we see that the $\tilde{c}$-segment (with respect to $q_{i}$ )

$$
[0,1] \ni t \mapsto \bar{z}_{i}(t)=\tilde{c}-\operatorname{Exp}_{q_{i}}\left((1-t) t_{i} p_{i}\right)=c-\operatorname{Exp}_{x_{i}}\left((1-t) \eta\left(t_{i} p_{i}\right)\right),
$$

from $\bar{z}_{i}(0)=\bar{z}_{i}$ to $\bar{z}_{i}(1)=\bar{x}_{\varepsilon, \delta}$, is of the form

$$
\bar{z}_{i}(t)=\left(\bar{x}_{\varepsilon, \delta}^{\prime}, \bar{z}_{i}^{\prime \prime}(t)\right) \in \bar{M}^{\prime} \times \bar{M}^{\prime \prime} .
$$

Observe that by Proposition 6.3 and Assumption 2.3, we have

$$
\bar{z}_{i}^{\prime \prime}(t) \in \bar{M}^{\prime \prime}\left(x^{\prime \prime}\right), \quad \forall t \in[0,1], \forall x \in Z_{\varepsilon, \delta, h} .
$$

We use these $\tilde{c}$-segments $\bar{z}_{i}(t)$ to define the points $\hat{p}_{i}, i=1, \ldots, n^{\prime \prime}$. Define the function

$$
m_{\bar{z}_{i}(t)}(q):=-\tilde{c}\left(q, \bar{z}_{i}(t)\right)+\tilde{c}\left(q_{i}, \bar{z}_{i}(t)\right)+\varphi\left(q_{i}\right) .
$$




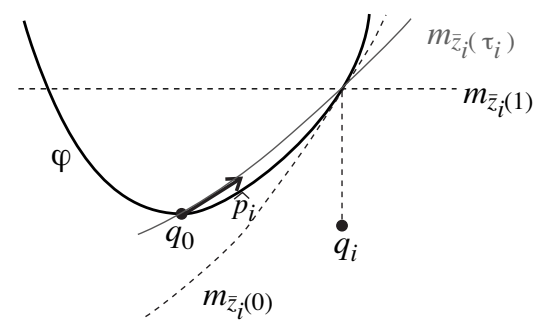

Fig. 4. The supporting function $m_{\bar{z}_{i}(0)}=m_{\bar{z}_{i}}$ touches $\varphi$ at $q_{i}$ from below. By interpolating between $m_{\bar{z}_{i}}=m_{\bar{z}_{i}(0)}$ and $m_{\bar{x}_{\varepsilon, \delta}}=m_{\bar{z}_{i}(1)}$ along the $\tilde{c}$-segment with respect to $q_{i}$, we can find $\tau_{i} \in[0,1)$ such that $m_{\bar{z}_{i}\left(\tau_{i}\right)}\left(q_{0}\right)=\varphi\left(q_{0}\right)$. Then the covector $\hat{p}_{i}$ used to construct $\tilde{C}$ is defined as $\hat{p}_{i}:=$ $\left(0,-D_{q^{\prime \prime}} \tilde{c}^{\prime \prime}\left(q_{0}^{\prime \prime}, \bar{z}_{i}^{\prime \prime}\left(\tau_{i}\right)\right) \in \partial m_{\bar{z}_{i}}\left(\tau_{i}\right)\left(q_{0}\right)\right.$.

Clearly, $m_{\bar{z}_{i}(0)} \leq \varphi$ and $m_{\bar{z}_{i}(1)} \equiv \varphi\left(q_{i}\right)=h+\varphi\left(q_{0}\right)$. By continuity there exists $\tau_{i} \in[0,1)$ such that

$$
m_{\bar{z}_{i}\left(\tau_{i}\right)}\left(q_{0}\right)=\varphi\left(q_{0}\right) .
$$

Also, Loeper's maximum principle (DASM) implies

$$
m_{\bar{z}_{i}\left(\tau_{i}\right)} \leq \max \left[h+\varphi\left(q_{0}\right), \varphi\right],
$$

so that in particular $m_{\bar{z}_{i}\left(\tau_{i}\right)} \leq \varphi$ on $\partial \tilde{Z}_{\varepsilon, \delta, h}$, hence, by the definition of $\left[\partial^{c} \phi\left(Z_{\varepsilon, \delta, h}\right)\right]_{x_{0}}$,

$$
\bar{z}_{i}\left(\tau_{i}\right) \in\left[\partial^{\tilde{c}} \varphi\left(\tilde{Z}_{\varepsilon, \delta, h}\right)\right]_{q_{0}}=\left[\partial^{c} \phi\left(Z_{\varepsilon, \delta, h}\right)\right]_{x_{0}} \quad \text { for every } i=1, \ldots, n^{\prime \prime} .
$$

For later use, consider the non-zero vectors

$$
\begin{aligned}
p_{i}\left(\tau_{i}\right) & =\left(1-\tau_{i}\right) t_{i} p_{i}=\left(0,\left(1-\tau_{i}\right) t_{i} p_{i}^{\prime \prime}\right) \\
& =\left(0,-D_{q^{\prime \prime}} \tilde{c}^{\prime \prime}\left(q_{i}^{\prime \prime}, \bar{z}_{i}^{\prime \prime}\left(\tau_{i}\right)\right)\right) \in T_{q_{i}^{\prime}}^{*}\left(T_{\bar{x}_{\varepsilon, \delta}^{\prime}}^{*} \bar{M}^{\prime}\right) \times T_{q_{i}^{\prime \prime}}^{*}\left(T_{\bar{x}_{\varepsilon, \delta}^{\prime \prime}}^{*} \bar{M}^{\prime \prime}\right), \quad i=1, \ldots, n^{\prime \prime} .
\end{aligned}
$$

Clearly these vectors are all mutually orthogonal. Moreover, because

$$
p_{i}\left(\tau_{i}\right) \in \partial m_{\bar{z}_{i}\left(\tau_{i}\right)}\left(q_{i}\right), \quad i=1, \ldots, n^{\prime \prime},
$$

by the convexity of $m_{\bar{z}_{i}}\left(\tau_{i}\right)$ we have

$$
\left|p_{i}\left(\tau_{i}\right)\right| \geq \frac{\varphi\left(q_{i}\right)-\varphi\left(q_{0}\right)}{\operatorname{dist}\left(q_{0}, \Pi_{i}\right)}=\frac{h}{\ell_{i}} .
$$

To finish the construction of $\tilde{C}$, let

$$
\hat{p}_{i}:=\left(0,-D_{q^{\prime \prime}} \tilde{c}^{\prime \prime}\left(q_{0}^{\prime \prime}, \bar{z}_{i}^{\prime \prime}\left(\tau_{i}\right)\right) \in T_{q_{0}^{\prime}}^{*}\left(T_{\bar{x}_{\varepsilon, \delta}^{\prime}}^{*} \bar{M}^{\prime}\right) \times T_{q_{0}^{\prime \prime}}^{*}\left(T_{\bar{x}_{\varepsilon, \delta}^{\prime \prime}}^{*} \bar{M}^{\prime \prime}\right), \quad i=1, \ldots, n^{\prime \prime} .\right.
$$

Notice that $\bar{z}_{i}\left(\tau_{i}\right)=\left(x_{\varepsilon, \delta}^{\prime}, \bar{z}_{i}\left(\tau_{i}\right)^{\prime \prime}\right)=\tilde{c}$-Exp $\operatorname{Ex}_{0} \hat{p}_{i}$. Let $\tilde{C}=\operatorname{co}\left(\hat{p}_{1}, \ldots, \hat{p}_{n^{\prime \prime}}\right)$ be the convex hull of $\hat{p}_{1}, \ldots, \hat{p}_{n^{\prime \prime}}$. We will see that $\tilde{C}$ has the desired properties (1)-(3). First, (1) follows immediately from (6.22), while (2) is a direct consequence of (6.19) and Lemma 2.8. 
Now, let us show (3). By (6.18) each $\bar{z}_{i}\left(\tau_{i}\right)^{\prime \prime}$ stays uniformly away (for small $\delta, h$ ) from the cut-locus of $\pi^{\prime \prime}\left(Z_{\varepsilon, \delta, h}\right)$. Hence we can apply Lemma 3.5 to (6.20) and (6.22) to see that $\hat{p}_{i}$ is close to $p_{i}\left(\tau_{i}\right)$ when we use the canonical identification $T_{q_{i}}^{*}\left(T_{x_{\varepsilon, \delta}}^{*} \bar{M}\right) \approx$ $T_{q_{0}}^{*}\left(T_{x_{\varepsilon, \delta}^{*}}^{*} \bar{M}\right)$; more precisely,

$$
\left|\hat{p}_{i}-p_{i}\left(\tau_{i}\right)\right| \leq o_{h}(1)\left|\hat{p}_{i}\right|
$$

where $o_{h}(1)$ is a quantity which goes to 0 as $h \rightarrow 0$. Since the vectors $\left\{p_{i}\left(\tau_{i}\right)\right\}_{i=1, \ldots, n^{\prime \prime}}$ are all mutually orthogonal, $\hat{p}_{i}$ are almost mutually orthogonal covectors, which by $(6.21)$ satisfy

$$
\left|\hat{p}_{i}\right|=\left|\hat{p}_{i}^{\prime \prime}\right| \gtrsim\left|p_{i}\left(\tau_{i}\right)\right| \geq h / \ell_{i} .
$$

(Here, for sufficiently small $\delta$ and $h / \delta$, the inequality $\gtrsim$ and the almost orthogonality are independent of $\delta, h$ and $\varepsilon$.) This gives

$$
\mathscr{H}^{n^{\prime \prime}}(\tilde{C}) \gtrsim \prod_{i=1}^{n^{\prime \prime}} \frac{h}{\ell_{i}} .
$$

This estimate combined with (6.17) shows (3), which completes the proof.

\subsection{Proof of Theorem 6.4: final argument}

In this section we finish the proof of Theorem 6.4. Let $0<\varepsilon<1$, and fix $0<\delta \leq$ $\delta_{1}(\varepsilon):=\min \left\{\delta_{0}(\varepsilon), \delta_{2}(\varepsilon)\right\}$ and $0<h \leq h_{1}(\varepsilon, \delta):=\min \left\{\delta^{2}, h_{2}(\varepsilon, \delta)\right\}$, with $\delta_{0}(\varepsilon)$ and $\delta_{2}(\varepsilon), h_{2}(\varepsilon, \delta)$ as in Propositions 6.3 and 6.7 respectively. The estimates $\lesssim, \gtrsim, \approx$ in this section are all independent of $\varepsilon, \delta$ and $h$.

To make use of the previous results, we need the following comparison result:

Proposition 6.9. The set $\tilde{C}$ constructed in Proposition 6.8 satisfies

$$
\mathscr{H}^{n^{\prime \prime}}(\tilde{C}) \lesssim\left|\partial^{c} \phi\left(Z_{\varepsilon, \delta, h}\right)\right|
$$

Note that even with Proposition 6.8(2), this estimate is not obvious because $n^{\prime \prime}<\operatorname{dim} M$.

Proof. For each $\varepsilon, \delta, h$ as in Proposition 6.8, we will find an auxiliary set $\mathcal{A}=\mathcal{A}_{\varepsilon, \delta, h} \subset$ $\mathcal{D}$ in a fixed (thus independent of $\varepsilon, \delta, h)$ compact set $\mathcal{D} \subset M^{*}\left(x_{0}\right) \subset T_{x_{0}}^{*} M$ such that

$$
\begin{aligned}
& c-\operatorname{Exp}_{x_{0}}(\mathcal{A}) \subset\left[\partial^{c} \phi\left(Z_{\varepsilon, \delta, h}\right)\right]_{x_{0}} \subset \partial^{c} \phi\left(Z_{\varepsilon, \delta, h}\right), \\
& |\mathcal{A}| \gtrsim \mathscr{H}^{n^{\prime \prime}}(\tilde{C}) .
\end{aligned}
$$

Once such a set is constructed, the desired estimate follows from

$$
\left|\partial^{c} \phi\left(Z_{\varepsilon, \delta, h}\right)\right| \geq\left|c-\operatorname{Exp}_{x_{0}}(\mathcal{A})\right| \gtrsim|\mathcal{A}| \quad(\text { since } \mathcal{A} \subset \mathcal{D})
$$


The construction of $\mathcal{A}$ goes through several steps. First, apply to the set $\tilde{C}$ the (extended) map $p \in T_{q_{0}}^{*}\left(T_{\bar{x}_{\varepsilon, \delta}}^{*} \bar{M}\right) \mapsto \eta(p) \in T_{x_{0}}^{*} M$ as in Lemma 3.4 (with $\bar{y}_{0}=\bar{x}_{\varepsilon, \delta}$ ), and let $\eta(\tilde{C}) \subset T_{x_{0}}^{*} M$ denote its image. Notice that by Proposition 6.8(2),

$$
c-\operatorname{Exp}_{x_{0}}(\eta(\tilde{C}))=\tilde{c}-\operatorname{Exp}_{q_{0}} \tilde{C} \subset\left[\partial^{c} \phi\left(Z_{\varepsilon, \delta, h}\right)\right]_{x_{0}} .
$$

Let us compare $\mathscr{H}^{n^{\prime \prime}}(\eta(\tilde{C}))$ with $\mathscr{H}^{n^{\prime \prime}}(\tilde{C})$. For each $p=\left(0, p^{\prime \prime}\right) \in \tilde{C}$, Lemma 3.4 applies as

$$
\eta(p)=\left(\eta_{\varepsilon, \delta}^{\prime}, p^{\prime \prime}\left(-D_{x^{\prime \prime}} D_{\bar{x}^{\prime \prime}} c^{\prime \prime}\left(x_{0}^{\prime \prime}, \bar{x}_{\varepsilon, \delta}^{\prime \prime}\right)\right)+\eta_{\varepsilon, \delta}^{\prime \prime}\right),
$$

where $\eta_{\varepsilon, \delta}=-D_{x} c\left(x_{0}, \bar{x}_{\varepsilon, \delta}\right)$ (thus, $c$ - $\left.\operatorname{Exp}_{x_{0}}\left(\eta_{\varepsilon, \delta}\right)=\bar{x}_{\varepsilon, \delta}\right)$. Therefore

$$
\eta(\tilde{C}) \subset\left\{\eta_{\varepsilon, \delta}^{\prime}\right\} \times T_{x_{0}^{\prime \prime}}^{*} M
$$

and

$$
\mathscr{H}^{n^{\prime \prime}}(\eta(\tilde{C}))=\left|\operatorname{det} D_{x^{\prime \prime}} D_{\bar{x}^{\prime \prime}} c^{\prime \prime}\left(x_{0}^{\prime \prime}, \bar{x}_{\varepsilon, \delta}^{\prime \prime}\right)\right| \mathscr{H}^{n^{\prime \prime}}(\tilde{C}) \approx \mathscr{H}^{n^{\prime \prime}}(\tilde{C}) .
$$

Notice that $\bar{x}_{\varepsilon, \delta}^{\prime \prime}$ is independent of $\varepsilon$ (see (6.7)) and stays uniformly away from $\operatorname{Cut}\left(x_{0}^{\prime \prime}\right)$, so that the above estimate is independent of $\varepsilon, \delta$ and $h$.

We now use a convexity argument to construct $\mathcal{A}$. We will first construct some suitable sets $C^{1}, \ldots, C^{b_{0}}$, and $\tilde{C}_{0}$, inside a fixed compact set (independent of $\left.\varepsilon, \delta, h\right)$ in $M^{*}\left(x_{0}\right)$, which have the properties of the sets $S_{i}$ in Lemma 3.3. These sets will also satisfy:

$$
\begin{aligned}
& c-\operatorname{Exp}_{x_{0}}\left(C^{1}\right) \cup \cdots \cup c-\operatorname{Exp}_{x_{0}}\left(C^{b_{0}}\right) \cup c-\operatorname{Exp}_{x_{0}}\left(\tilde{C}_{0}\right) \subset\left[\partial^{c} \phi\left(Z_{\varepsilon, \delta, h}\right)\right]_{x_{0}}, \\
& \mathscr{H}^{n_{i}}\left(C^{i}\right) \gtrsim 1, \quad i=1, \ldots, b_{0}, \\
& \mathscr{H}^{n^{\prime \prime}}\left(\tilde{C}_{0}\right) \gtrsim \mathscr{H}^{n^{\prime \prime}}(\eta(\tilde{C})) .
\end{aligned}
$$

Then $\mathcal{A}$ will be given as the convex hull $\operatorname{co}\left(C^{1}, \ldots, C^{b_{0}}, \tilde{C}_{0}\right)$. By convexity of $M^{*}\left(x_{0}\right)$, $\mathcal{A}$ will be in a fixed compact set, say $\mathcal{D}$, independent of $\varepsilon, \delta, h$, and the $c$-convexity of $\left[\partial^{c} \phi\left(Z_{\varepsilon, \delta, h}\right)\right]_{x_{0}}$ (see Lemma 2.8) will imply $c$ - $\operatorname{Exp}_{x_{0}}(\mathcal{A}) \subset\left[\partial^{c} \phi\left(Z_{\varepsilon, \delta, h}\right)\right]_{x_{0}}$, showing (6.23). We will then apply Lemma 3.3 to get

$$
|\mathcal{A}| \gtrsim \mathscr{H}^{n^{\prime \prime}}(\eta(\tilde{C})) \approx \mathscr{H}^{n^{\prime \prime}}(\tilde{C}),
$$

which gives (6.24). Hence we are led to construct $C^{1}, \ldots, C^{b_{0}}, \tilde{C}_{0}$.

To construct $C^{1}, \ldots, C^{b_{0}}$, recall that $M^{\prime}=M^{1} \times \cdots \times M^{b_{0}}$, and for every $i \in$ $\left\{1, \ldots, b_{0}\right\}$ there exists $\bar{y}_{i} \in \partial^{c} \phi\left(x_{0}\right)$ with $\bar{y}_{i}^{i}=-x_{0}^{i}$. Moreover $M_{\bar{y}_{i}}^{i} \subset \partial^{c} \phi\left(x_{0}\right)$ by Lemma 6.6. We further observe that the same inclusion holds for all the components $y_{i}^{l}$ of $y_{i}$ that satisfy $y_{i}^{l}=-x_{0}^{l}$. Hence, once $\bar{y}_{i}$ has a cut-locus component with $x_{0}$, one can change that component arbitrarily, and the resulting point still remains inside $\partial^{c} \phi\left(x_{0}\right)$. Combining this fact with Loeper's maximum principle (DASM) we can find a covector $v_{i}$ and a set $C^{i} \subset \partial \phi\left(x_{0}\right) \subset T_{x_{0}}^{*} M$, with $v_{i} \in C^{i}$ whose components are either $v_{i}^{l}=0$ or $v_{i}^{l} \in M^{l *}\left(x_{0}^{l}\right)$, and

$$
C^{i}=\left\{q \in T_{x_{0}}^{*} M \mid 2 q^{i} \in M^{i *}\left(x_{0}^{i}\right), \text { and } q^{l}=v_{i}^{l} \text { for } l \neq i\right\} .
$$


Clearly, $C^{i}$ is compact and $C^{i} \subset M^{*}\left(x_{0}\right)$. Moreover $c$-Exp $x_{x_{0}} C^{i} \subset \partial^{c} \phi\left(x_{0}\right) \subset$ $\left[\partial^{c} \phi\left(x_{0}\right)\right]_{x_{0}}$ and $\mathscr{H}^{n_{i}}\left(C^{i}\right) \gtrsim 1$. Also, observe that the construction of $C^{1}, \ldots, C^{b_{0}}$ is independent of $\varepsilon, \delta, h$.

Let us now construct the set $\tilde{C}_{0}$. From Propositions 6.2 and 6.3 we see that for $\delta$ and $h / \delta$ sufficiently small there exists a compact set $C^{\prime \prime} \subset \bar{M}^{\prime \prime}\left(x_{0}^{\prime \prime}\right)$ (independent of $\varepsilon, \delta, h$ ) with $\pi^{\prime \prime}\left(\partial^{c} \phi\left(Z_{\varepsilon, \delta, h}\right)\right) \subset C^{\prime \prime}$. Recall the definition of $a_{0}, b_{0}, \bar{x}_{\varepsilon, \delta}=\left(\bar{x}_{\varepsilon}^{\cdot}, \bar{x}_{\delta}^{\cdot \cdot}\right)$, and that

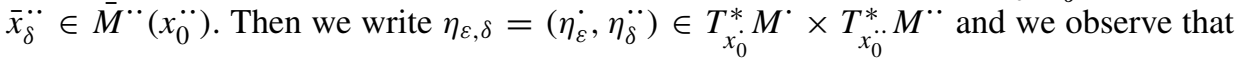
$\eta_{\delta}$ is uniformly away from the boundary of $M^{* *}\left(x_{0}^{\ddot{*}}\right)$. These facts imply that there exists a compact set $C_{2}^{\cdot \bullet} \subset M^{* *}\left(x_{0}^{* *}\right)$ (independent of $\left.\varepsilon, \delta, h\right)$ such that

$$
\eta(\tilde{C}) \subset\left\{\eta_{\varepsilon}^{\cdot}\right\} \times C_{2}^{*} .
$$

However, $\eta_{\dot{\varepsilon}}^{\cdot} \rightarrow \partial M^{* *}\left(x_{0}^{\cdot}\right)$ as $\varepsilon \rightarrow 0$, thus $\eta(\tilde{C})$ is not kept in a fixed compact set in $M^{*}\left(x_{0}\right)$. In particular, we cannot take $\eta(\tilde{C})$ for $\tilde{C}_{0}$, and this motivates the following: Since $\bar{x}_{\varepsilon}^{\cdot}=-x_{0}^{\cdot}$ and $\bar{x}_{\varepsilon, \delta} \in\left[\partial^{c} \phi\left(Z_{\varepsilon, \delta, h}\right)\right]_{x_{0}}$, applying Lemma 6.6 as in the previous paragraph we see that the set $M \times\left\{\bar{x}_{\delta}^{\cdot \cdot}\right\}$, in particular $\left(x_{0}^{\cdot}, \bar{x}_{\delta}^{\cdot \cdot}\right)$, belongs to $\left[\partial^{c} \phi\left(Z_{\varepsilon, \delta, h}\right)\right]_{x_{0}}$. The point $\left(x_{0}^{\cdot}, \bar{x}_{\delta}^{\cdot \cdot}\right)$ corresponds to the covector $\left(0, \eta_{\delta}^{\cdot \ddot{ }}\right)$. Consider the cone $\operatorname{co}\left(\left(0, \eta_{\delta}^{\ddot{*}}\right), \eta(\tilde{C})\right)$, and define

$$
\tilde{C}_{0}:=\operatorname{co}\left(\left(0, \ddot{\eta_{\delta}}\right), \eta(\tilde{C})\right) \cap\left\{\left(\eta_{\varepsilon}^{\cdot} / 2, q^{\cdot *}\right) \in T_{x_{0}}^{*} M \mid q^{\cdot} \in T_{x_{0}^{*}}^{*} M^{*}\right\} .
$$

By a simple geometric argument

$$
\mathscr{H}^{n^{\prime \prime}}\left(\tilde{C}_{0}\right) \gtrsim \mathscr{H}^{n^{\prime \prime}}(\eta(\tilde{C})),
$$

and moreover, since $\eta_{\varepsilon}^{\cdot} / 2 \in \frac{1}{2} M^{* *}\left(x_{0}^{\cdot}\right)$, the set $\tilde{C}_{0}$ is contained in a fixed compact set in $M^{*}\left(x_{0}\right)$ independently of $\varepsilon, \delta, h$. By $c$-convexity of $\left[\partial^{c} \phi\left(Z_{\varepsilon, \delta, h}\right)\right]_{x_{0}}$,

$$
c-\operatorname{Exp}_{x_{0}}\left(\tilde{C}_{0}\right) \subset\left[\partial^{c} \phi\left(Z_{\varepsilon, \delta, h}\right)\right]_{x_{0}} .
$$

Note that by construction this set $\tilde{C}_{0}$, together with $C^{1}, \ldots, C^{b_{0}}$, have the properties of the sets $S_{i}$ in Lemma 3.3. Furthermore they are in a fixed compact set in $M^{*}\left(x_{0}\right)$ independent of $\varepsilon, \delta, h$. This completes the proof.

Combining Propositions 6.9 and 6.8(3) we obtain

$$
h^{n^{\prime \prime}} \lesssim \mathscr{H}^{n^{\prime \prime}}\left(\pi^{\prime \prime}\left(\tilde{Z}_{\varepsilon, \delta, h}\right)\right)\left|\partial^{c} \phi\left(Z_{\varepsilon, \delta, h}\right)\right|
$$

We will finish the proof by applying Proposition 6.7. First, we need some preliminary steps. Using the notation of Section 6.5 , let $Z_{\varepsilon, \delta, h}^{\prime}$ be the slice of $Z_{\varepsilon, \delta, h}$ in $M^{\prime} \times\left\{x_{0}^{\prime \prime}\right\}$, that is,

$$
Z_{\varepsilon, \delta, h}^{\prime}:=\left\{x^{\prime} \in M^{\prime} \mid\left(x^{\prime}, x_{0}^{\prime \prime}\right) \in Z_{\varepsilon, \delta, h}\right\} .
$$

Then $Z_{\varepsilon, \delta, h}^{\prime}$ is embedded via $x^{\prime} \mapsto-D_{\bar{x}^{\prime}} c^{\prime}\left(x^{\prime}, \bar{x}_{\varepsilon, \delta}^{\prime}\right)$ into $\tilde{Z}_{\varepsilon, \delta, h}^{\prime} \subset \bar{M}^{* *}\left(\bar{x}_{\varepsilon, \delta}^{\prime}\right)$, where

$$
\tilde{Z}_{\varepsilon, \delta, h}^{\prime}:=\left\{q^{\prime} \in \bar{M}^{\prime *}\left(\bar{x}_{\varepsilon, \delta}^{\prime}\right) \mid\left(q^{\prime}, q_{0}^{\prime \prime}\right) \in \tilde{Z}_{\varepsilon, \delta, h}\right\} .
$$

Embed in the same way each $Z_{\varepsilon, \delta, h}^{i}(\operatorname{see}(6.13)), i=1, \ldots, b_{0}$, into $\tilde{Z}_{\varepsilon, \delta, h}^{i} \subset \bar{M}^{i *}\left(\bar{x}_{\varepsilon, \delta}^{i}\right)$. 
Proposition 6.10. Assume that $0<\delta \leq \delta_{0}$ and $0<h \leq \delta^{2}$, with $\delta_{0}$ as in Proposition 6.3.

Then

$$
\begin{aligned}
\left(\min _{x^{\prime} \in Z_{\varepsilon, \delta, h}^{\prime}}\left|\operatorname{det}\left(D_{x^{\prime}} D_{\bar{x}^{\prime}} c^{\prime}\left(x^{\prime}, \bar{x}_{\varepsilon, \delta}^{\prime}\right)\right)\right|\right)\left|Z_{\varepsilon, \delta, h}^{\prime}\right| & \leq\left|\tilde{Z}_{\varepsilon, \delta, h}^{\prime}\right| \\
& \leq\left(\max _{x^{\prime} \in Z_{\varepsilon, \delta, h}^{\prime}}\left|\operatorname{det}\left(D_{x^{\prime}} D_{\bar{x}^{\prime}} c^{\prime}\left(x^{\prime}, \bar{x}_{\varepsilon, \delta}^{\prime}\right)\right)\right|\right)\left|Z_{\varepsilon, \delta, h}^{\prime}\right|, \\
\left(\min _{x^{i} \in Z_{\varepsilon, \delta, h}^{i}}\left|\operatorname{det}\left(D_{x^{i}} D_{\bar{x}^{i}} c^{i}\left(x^{i}, \bar{x}_{\varepsilon, \delta}^{i}\right)\right)\right|\right)\left|Z_{\varepsilon, \delta, h}^{i}\right| & \leq\left|\tilde{Z}_{\varepsilon, \delta, h}^{i}\right| \\
& \leq\left(\max _{x^{i} \in Z_{\varepsilon, \delta, h}^{i}}\left|\operatorname{det}\left(D_{x^{i}} D_{\bar{x}^{i}} c^{i}\left(x^{i}, \bar{x}_{\varepsilon, \delta}^{i}\right)\right)\right|\right)\left|Z_{\varepsilon, \delta, h}^{i}\right|,
\end{aligned}
$$

where $|\cdot|$ denotes the Riemannian volume (in the appropriate submanifold).

Proof. From (6.14),

$$
D_{x^{\prime}} q^{\prime}=-D_{x^{\prime}} D_{\bar{x}^{\prime}} c^{\prime}\left(x^{\prime}\left(q^{\prime}\right), \bar{x}_{\varepsilon, \delta}^{\prime}\right),
$$

and so the first inequality follows from

$$
\left|\tilde{Z}_{\varepsilon, \delta, h}^{\prime}\right|=\int_{Z_{\varepsilon, \delta, h}^{\prime}}\left|\operatorname{det} D_{x^{\prime}} q^{\prime}\right| d x^{\prime} .
$$

The proof of the second inequality is analogous.

By convexity and Lemma 3.3 one has

$$
\mathscr{H}^{n^{\prime}}\left(\tilde{Z}_{\varepsilon, \delta, h}^{\prime}\right) \gtrsim \prod_{i=1}^{b_{0}} \mathscr{H}^{n_{i}}\left(\tilde{Z}_{\varepsilon, \delta, h}^{i}\right),
$$

while Propositions 6.10 and 6.7 imply

$$
\begin{aligned}
\prod_{i=1}^{b_{0}} \mathscr{H}^{n_{i}}\left(\tilde{Z}_{\varepsilon, \delta, h}^{i}\right) & \geq \prod_{i=1}^{b_{0}}\left(\min _{x^{i} \in Z_{\varepsilon, \delta, h}^{i}}\left|\operatorname{det}\left(D_{x^{i}} D_{\bar{x}^{i}} c^{i}\left(x^{i}, \bar{x}_{\varepsilon, \delta}^{i}\right)\right)\right|\right)\left|Z_{\varepsilon, \delta, h}^{i}\right| \\
& \gtrsim\left[\prod_{i=1}^{b_{0}}\left(\min _{x^{i} \in Z_{\varepsilon, \delta, h}^{i}}\left|\operatorname{det}\left(D_{x^{i}} D_{\bar{x}^{i}} c^{i}\left(x^{i}, \bar{x}_{\varepsilon, \delta}^{i}\right)\right)\right|\right)\right] \frac{h^{n^{\prime}}}{\varepsilon^{a_{0}}}
\end{aligned}
$$

Combining these estimates with (6.25) we get

$$
\begin{aligned}
& h^{n^{\prime}+n^{\prime \prime}} \lesssim \varepsilon^{a_{0}}\left[\prod_{i=1}^{b_{0}} \min _{x^{i} \in Z_{\varepsilon, \delta, h}^{i}}\left|\operatorname{det}\left(D_{x^{i}} D_{\bar{x}^{i}} c^{i}\left(x^{i}, \bar{x}_{\varepsilon, \delta}^{i}\right)\right)\right|\right]^{-1} \\
& \mathscr{H}^{n^{\prime}}\left(\tilde{Z}_{\varepsilon, \delta, h}^{\prime}\right) \mathscr{H}^{n^{\prime \prime}}\left(\pi^{\prime \prime}\left(\tilde{Z}_{\varepsilon, \delta, h}\right)\right)\left|\partial^{c} \phi\left(Z_{\varepsilon, \delta, h}\right)\right| \\
& \lesssim \varepsilon^{a_{0}}\left[\prod_{i=1}^{b_{0}} \min _{x^{i} \in Z_{\varepsilon, \delta, h}^{i}}\left|\operatorname{det}\left(D_{x^{i}} D_{\bar{x}^{i}} c^{i}\left(x^{i}, \bar{x}_{\varepsilon, \delta}^{i}\right)\right)\right|\right]^{-1}\left|\tilde{Z}_{\varepsilon, \delta, h}\right|\left|\partial^{c} \phi\left(Z_{\varepsilon, \delta, h}\right)\right| \quad \text { (by Lemma 3.2) } \\
& \lesssim \varepsilon^{a_{0}}\left|Z_{\varepsilon, \delta, h}\right|\left|\partial^{c} \phi\left(Z_{\varepsilon, \delta, h}\right)\right|,
\end{aligned}
$$


where the last inequality follows from

$$
\left|\tilde{Z}_{\varepsilon, \delta, h}\right| \lesssim\left(\max _{x \in Z_{\varepsilon, \delta, h}} \operatorname{det}\left(D_{x} D_{\bar{x}} c\left(x, \bar{x}_{\varepsilon, \delta}\right)\right)\right)\left|Z_{\varepsilon, \delta, h}\right|
$$

(see Proposition 6.10) and

$$
\frac{\max _{x^{\prime} \in Z_{\varepsilon, \delta, h}^{\prime}} \operatorname{det}\left(D_{x^{\prime}} D_{\bar{x}^{\prime}} c^{\prime}\left(x^{\prime}, \bar{x}_{\varepsilon, \delta}^{\prime}\right)\right)}{\prod_{i=1}^{b_{0}} \min _{x^{i} \in Z_{\varepsilon, \delta, h}^{i}}\left|\operatorname{det}\left(D_{x^{i}} D_{\bar{x}^{i}} c^{i}\left(x^{i}, \bar{x}_{\varepsilon, \delta}^{i}\right)\right)\right|} \lesssim 1 \quad \text { as } \delta, h / \delta \rightarrow 0
$$

(see Propositions 6.2 and 6.3). This concludes the proof of Theorem 6.4, and Theorem 5.1 is proved.

Acknowledgments. The authors are pleased to thank Neil Trudinger, Tom Spencer, and Cédric Villani for useful discussions.

The authors are grateful to the Institute for Pure and Applied Mathematics at UCLA and the Institute for Advanced Study in Princeton for their generous hospitality during various stages of this work. AF is partially supported by NSF grant DMS-0969962. RJM is supported in part by NSERC grants 217006-08 and NSF grant DMS-0354729. YHK is supported partly by NSF grant DMS-0635607 through the membership at Institute for Advanced Study at Princeton NJ, and also in part by NSERC grant 371642-09. Any opinions, findings and conclusions or recommendations expressed in this material are those of the authors and do not reflect the views of either the Natural Sciences and Engineering Research Council of Canada (NSERC) or the United States National Science Foundation (NSF).

\section{References}

[Br] Brenier, Y:: Polar factorization and monotone rearrangement of vector-valued functions. Comm. Pure Appl. Math. 44, 375-417 (1991) Zbl 0738.46011 MR 1100809

[Ca1] Caffarelli, L. A.: A localization property of viscosity solutions to the Monge-Ampère equation and their strict convexity. Ann. of Math. 131, 129-134 (1990) Zbl 0704.35045 MR 1038359

[Ca2] Caffarelli, L. A.: Some regularity properties of solutions of Monge Ampère equation. Comm. Pure Appl. Math. 44, 965-969 (1991) Zbl 0761.35028 MR 1127042

[Ca3] Caffarelli, L. A.: The regularity of mappings with a convex potential. J. Amer. Math. Soc. 5, 99-104 (1992) Zbl 0753.35031 MR 1124980

[Ca4] Caffarelli, L. A.: Boundary regularity of maps with convex potentials II. Ann. of Math. 144, 453-496 (1996) Zbl 0916.35016 MR 1426885

[Ca5] Caffarelli, L. A.: Monotonicity properties of optimal transportation and the FKG and related inequalities. Comm. Math. Phys. 214, 547-563 (2000) Zbl 0978.60107 MR 1800860

[Co] Cordero-Erausquin, D.: Sur le transport de mesures périodiques. C. R. Acad. Sci. Paris Sér. I Math. 329, 199-202 (1999) Z Zbl 0942.28015 MR 1711060

[D1] Delanoë, P.: Classical solvability in dimension two of the second boundary value problem associated with the Monge-Ampère operator. Ann. Inst. Henri Poincaré Anal. Non Linéaire 8, 443-457 (1991) Zbl 0778.35037 MR 1136351

[D2] Delanoë, P.: Gradient rearrangement for diffeomorphisms of a compact manifold. Differential Geom. Appl. 20, 145-165 (2004) Zbl 1039.58008 MR 2038552 
[DG] Delanoë, P., Ge, Y.: Regularity of optimal transportation maps on compact, locally nearly spherical, manifolds. J. Reine Angew. Math. 646, 65-115 (2010) Zbl 1200.58025 MR 2719556

[DL] Delanoë, P., Loeper, G.: Gradient estimates for potentials of invertible gradient mappings on the sphere. Calc. Var. Partial Differential Equations 26, 297-311 (2006) Zbl 1136.35358 MR 2232207

[FF] Fathi, A., Figalli, A.: Optimal transportation on non-compact manifolds. Israel J. Math. 175, 1-59 (2010) Zbl 1198.49044 MR 2607536

[F] Figalli, A.: Existence, uniqueness, and regularity of optimal transport maps. SIAM J. Math. Anal. 39, 126-137 (2007) Zbl 1132.28322 MR 2318378

[FKM1] Figalli, A., Kim, Y.-H., McCann, R. J.: Hölder continuity and injectivity of optimal maps. Arch. Ration. Mech. Anal., to appear

[FKM2] Figalli, A., Kim, Y.-H., McCann, R. J.: When is a multidimensional screening a convex program? J. Econom. Theory 146, 454-478 (2011) Zbl pre05882918 MR 2888826

[FR] Figalli, A., Rifford, L.: Continuity of optimal transport maps on small deformations of $\mathbb{S}^{2}$. Comm. Pure Appl. Math. 62, 1670-1706 (2009) Zbl 1175.49040 MR 2569074

[FRV] Figalli, A., Rifford, L., Villani, C.: Necessary and sufficient conditions for continuity of optimal transport maps on Riemannian manifolds. Tohoku Math. J. 63, 855-876 (2011) Zbl pre06016717 MR 2872966

[FV] Figalli, A., Villani, C.: An approximation lemma about the cut locus, with applications in optimal transport theory. Methods Appl. Anal. 15, 149-154 (2008) Zbl 1172.53022 MR 2481676

[G] Gutiérrez, C.: The Monge-Ampère Equation. Birkhäuser (2001) Zbl 0989.35052 MR 1829162

[J] John, F.: Extremum problems with inequalities as subsidiary conditions. In: Studies and Essays Presented to R. Courant on his 60th Birthday, January 8, 1948, Interscience Publ., New York, 187-204 (1948) Zbl 0034.10503 MR 0030135

[K] Kim, Y.-H.: Counterexamples to continuity of optimal transportation on positively curved Riemannian manifolds. Int. Math. Res. Notices 2008, art. ID rnn120, 15 pp. Zbl 1160.49047 MR 2448078

[KM1] Kim, Y.-H., McCann, R. J.: Continuity, curvature, and the general covariance of optimal transportation. J. Eur. Math. Soc. 12, 1009-1040 (2010) Zbl 1191.49046 MR 2654086

[KM1a] Kim, Y.-H., McCann, R. J.: Appendices to original version of "Continuity, curvature, and the general covariance of optimal transportation". arXiv:math/0712.3077v1

[KM2] Kim, Y.-H., McCann, R. J.: Towards the smoothness of optimal maps on Riemannian submersions and Riemannian products (of round spheres in particular). J. Reine Angew. Math. 664, 1-27 (2012) Zbl 1239.53049 MR 2980128

[L] Levin, V. L.: Abstract cyclical monotonicity and Monge solutions for the general MongeKantorovich problem. Set-Valued Anal. 7, 7-32 (1999) Zbl 0934.54013 MR 1699061

[L1] Loeper, G.: On the regularity of solutions of optimal transportation problems. Acta Math. 202, 241-283 (2009) Zbl 1219.49038 MR 2506751

[L2] Loeper, G.: Regularity of optimal maps on the sphere: the quadratic cost and the reflector antenna. Arch. Ration. Mech. Anal. 199, 269-289 (2011) Zbl 1231.35280 MR 2754343

[LV] Loeper, G., Villani, C.: Regularity of optimal transport in curved geometry: the nonfocal case. Duke Math. J. 151, 431-485 (2010) Zbl 1192.53041 MR 2605867

[Li] Liu, J., Hölder regularity of optimal mappings in optimal transportation. Calc. Var. Partial Differential Equations 34, 435-451 (2009) Zbl 1166.35331 MR 2476419 
[M] McCann, R. J.: Polar factorization of maps on Riemannian manifolds. Geom. Funct. Anal. 11, 589-608 (2001) Zbl 1011.58009 MR 1844080

[LTW] Liu, J., Trudinger, N., Wang, X.-J.: Interior $C^{2, \alpha}$-regularity for potential functions in optimal transportation. Comm. Partial Differential Equations 35, 165-184 (2010) Zbl 1189.35142 MR 2748621

[MTW] Ma, X.-N., Trudinger, N., Wang, X.-J.: Regularity of potential functions of the optimal transport problem. Arch. Ration. Mech. Anal. 177, 151-183 (2005) Zbl 1072.49035 MR 2188047

[S] Sei, T.: A Jacobian inequality for gradient maps on the sphere and its application to directional statistics. Comm. Statist. Theory Methods, to appear

[TW] Trudinger, N., Wang, X.-J.: On the second boundary value problem for Monge-Ampère type equations and optimal transportation. Ann. Scuola Norm. Sup. Pisa Cl. Sci. (5) 8, 143-174 (2009) Zbl 1182.35134 MR 2512204

[U] Urbas, J.: On the second boundary value problem for equations of Monge-Ampère type. J. Reine Angew. Math. 487, 115-124 (1997) Zbl 0880.35031 MR 1454261

[V] Villani, C.: Optimal Transport, Old and New. Grundlehren Math. Wiss. 338, Springer, Berlin (2009) Zbl 1156.53003 MR 2459454 